\title{
Enhancing Semantic Bidirectionalization via Shape Bidirectionalizer Plug-ins
}

\author{
JANIS VOIGTLÄNDER \\ University of Bonn \\ and \\ ZHENJIANG HU \\ National Institute of Informatics, Tokyo \\ and \\ KAZUTAKA MATSUDA \\ University of Tokyo \\ and \\ MENG WANG \\ Chalmers University of Technology
}

\begin{abstract}
Matsuda et al. (2007) and Voigtländer (2009) have introduced two techniques that given a sourceto-view function provide an update propagation function mapping an original source and an updated view back to an updated source, subject to standard consistency conditions. Previously, we developed a synthesis of the two techniques, based on a separation of shape and content aspects (Voigtländer et al.2010). Here, we carry that idea further, reworking the technique of Voigtländer such that any shape bidirectionalizer (based on the work of Matsuda et al. or not) can be used as a plug-in, to good effect. We also provide a data-type-generic account, enabling wider reuse, including the use of pluggable bidirectionalization itself as a plug-in.
\end{abstract}

\section{Introduction}

Bidirectionalization is the task of, given some function get $:: \tau_{1} \rightarrow \tau_{2}$, producing a function put $:: \tau_{1} \rightarrow \tau_{2} \rightarrow \tau_{1}$ such that if get maps an original source $s$ to an original view $v$, and $v$ is somehow changed into an updated view $v^{\prime}$, then put applied to $s$ and $v^{\prime}$ produces an updated source $s^{\prime}$ in a meaningful way.

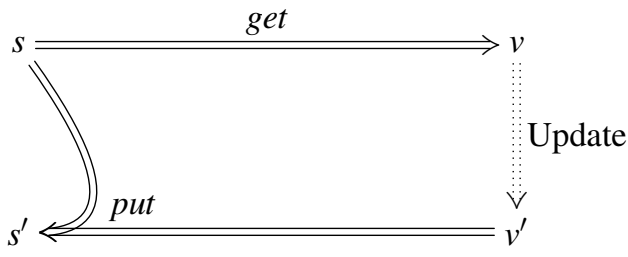

Such get/put-pairs, called bidirectional transformations, play an important role in various application areas such as databases, file synchronization, structured editing, and model transformation. Czarnecki et al. (2009) survey relevant techniques and open problems. 
Functional programming approaches have had an important impact, with several ideas and solutions springing from this part of the programming languages field in particular (Bohannon et al. 2006, 2008, Foster et al. 2007, 2008, 2012; Hidaka et al.|2010, Hu et al. 2008; Matsuda and Wang 2013, Matsuda et al. 2007, 2009, Pacheco and Cunha 2010, Pacheco et al. 2012, Voigtländer 2009, 2012; Wang et al. 2011, 2012).

Automatic bidirectionalization is one approach to obtaining suitable get/put-pairs; others are domain-specific languages or more ad-hoc programming techniques. Two different flavors of bidirectionalization have been proposed: syntactic and semantic. Syntactic bidirectionalization (Matsuda et al. 2007) works on a syntactic representation of (somehow restricted) get-functions and synthesizes appropriate definitions for $p u t$-functions algorithmically. Semantic bidirectionalization (Voigtländer 2009) does not inspect the syntactic definitions of $g e t$-functions at all, but instead provides a single definition of put, parameterized over get as a semantic object, that does the job by invoking get in a kind of "simulation mode".

Both syntactic and semantic bidirectionalization have their strengths and weaknesses. Syntactic bidirectionalization heavily depends on syntactic restraints exercised when implementing the get-function. Basically, the technique of Matsuda et al. (2007) can only deal with programs in a custom first-order language subject to certain restrictions concerning variable use and nested function calls. Semantic bidirectionalization, in contrast, provides very easy access to bidirectionality within a general-purpose language, liberated from the syntactic corset as to how to write functions of interest. The price to pay for this in the case of the approach of Voigtländer (2009) is that it works for polymorphic functions only, and in the original form is unable to deal with view updates that change the shape of a data structure. The syntactic approach, on the other hand, is successful for many such shapechanging updates, and can deal with non-polymorphic functions.

Voigtländer et al. (2010) developed an approach for combining syntactic and semantic bidirectionalization. The resulting technique inherits the limitations in program coverage from both techniques, but gains improved updateability: more $\left(s, v^{\prime}\right)$ pairs can successfully be mapped to a suitable $s^{\prime}$ by put (see the next section for a more formal conceptualization). Specifically, semantic bidirectionalization now gets a chance to deal with shape-changing updates, and the combined technique is superior to syntactic bidirectionalization on its own in many cases (and actually never worse than the better of the two original techniques). The combination strategy we pursued was essentially motivated by combining the specialties of the two approaches. Semantic bidirectionalization's specialty is to employ polymorphism to deal with the content elements of data structures in a very lightweight way. In fact, in the original technique, the shape and content aspects of a data structure are completely separated, updates affecting the shape are completely outlawed, arbitrary updates to content elements can be simply absorbed, and by recombining original shape with updated content the desired update consistency is guaranteed. Syntactic bidirectionalization's specialty is to have a more refined, and case-by-case, notion of what updates, including updates on the shape aspect, can be permitted. But it turns out that content elements often get in the way. In fact, by having to deal with both shape and content, at the same time, in the key step of syntactic bidirectionalization (namely "view complement derivation"), updateability is hampered. In our combined approach we divided the labor: semantic bidirectionalization deals with content only, syntactic bidirectionalization deals with shape only. As a result, 
the reach of semantic bidirectionalization is expanded beyond shape-preserving updates, and syntactic bidirectionalization is invoked on a more specialized kind of program, on which it can yield better results, benefitting both.

In the current paper, we carry the idea further: since the combined approach essentially treats syntactic bidirectionalization as a black box, we can consider it as a completely external component, and indeed replace it by other approaches (than that of Matsuda et al.) for obtaining bidirectional transformations on shapes. Any such approach can be "plugged into" the semantic technique (after suitable dissection/refactoring of the latter). We develop the details and consider some such plug-ins, including (in Section 5.3) of course the specific use case of combining the techniques of Matsuda et al. (2007) and Voigtländer (2009), thus covering the results of Voigtländer et al. (2010). We also generalize from lists (to which case the development of Voigtländer et al. was restricted) to more general data types (in Section 7). As a bonus, this enables a kind of bootstrapping in which pluggable bidirectionalization is itself used as a plug-in.

\section{Consistency Conditions and Language Setup}

To explain what we mean by improved updateability, we have to elaborate on the phrase "in a meaningful way" in the first sentence of the introduction, and on "suitable" at the start of its second paragraph. So, when is a get/put-pair "good"? How should $s, v, v^{\prime}$, and $s^{\prime}$ in get $s \equiv v$ and put $s v^{\prime} \equiv s^{\prime}$ be related? One natural requirement is that if $v \equiv v^{\prime}$, then $s \equiv s^{\prime}$, or, put differently,

$$
\text { put } s(\text { get } s) \equiv s \text {. }
$$

Another requirement to expect is that $s^{\prime}$ and $v^{\prime}$ should be related in the same way as $s$ and $v$ are, or, again expressed as a round-trip property,

$$
\text { get }\left(\text { put } s v^{\prime}\right) \equiv v^{\prime} \text {. }
$$

These are the standard consistency conditions (Bancilhon and Spyratos 1981) known as GetPut and PutGet (Foster et al.2007). But the latter of the two is often too hard to satisfy in practice. For fixed get, it can be impossible to provide a put-function fulfilling equation (2) for every choice of $s$ and $v^{\prime}$, simply because $v^{\prime}$ may not even be in the range of get. One solution is to make the put-function partial and to only expect the PutGet law to hold in case put $s v^{\prime}$ is actually defined. Of course, a trivially consistent put-function we could then always come up with is the one for which put $s v^{\prime}$ is only defined if get $s \equiv v^{\prime}$ and which simply returns $s$ then. Clearly, this choice would satisfy both equations (1) and (2), but would be utterly useless in terms of updateability. The very idea that $v$ and $v^{\prime}$ can be different in the original scenario would be countermanded.

So our evaluation criteria for "goodness" are that get/put should satisfy equation (11), that they should satisfy equation (2) whenever put $s v^{\prime}$ is defined, and that put $s v^{\prime}$ should be actually defined on a big part of its potential domain, indeed preferably for all $s$ and $v^{\prime}$ of appropriate type. That measure, simply comparing the sizes of the applicability domains of put, is somewhat coarse, but we will also discuss finer distinctions (i.e., concerning what two different put-functions map a given $\left(s, v^{\prime}\right)$ pair to) later.

Since our emphasis is on the updateability inherent in a get/put-pair, we make the partiality of put explicit in the type (and make the function itself total) via optionality 
of the return value, using the data type definition

$$
\text { data Maybe } \alpha=\text { Nothing } \mid \text { Just } \alpha
$$

The following definition formulates the consistency conditions for this setting.

Definition 1. Let $\tau_{1}$ and $\tau_{2}$ be types. Let functions get $:: \tau_{1} \rightarrow \tau_{2}$ and put :: $\tau_{1} \rightarrow \tau_{2} \rightarrow$ Maybe $\tau_{1}$ be given. We say that put is consistent for get if:

- For every $s:: \tau_{1}$, put $s$ (get $\left.s\right) \equiv$ Just $s$.

- For every $s, s^{\prime}:: \tau_{1}$ and $v^{\prime}:: \tau_{2}$, if put $s v^{\prime} \equiv$ Just $s^{\prime}$, then get $s^{\prime} \equiv v^{\prime}$.

We work in Haskell (Peyton Jones 2003) with a few GHC extensions, almost: one deviation we make is that we assume that the type Int is replaced, throughout the language, by a type Nat, discarding all negative integers. In particular, the function length on lists will have type $[\alpha] \rightarrow$ Nat instead of $[\alpha] \rightarrow \operatorname{Int}$, and we assume we are given a variant Data.NatMap of standard module Data.IntMap. We use $\equiv$ for semantic equivalence, but specialize notation to $=$ for natural numbers. All functions, from now on, are assumed to be total, except where partiality is explicitly mentioned.

\section{The Original Semantic Bidirectionalization Technique}

We briefly introduce the technique of Voigtländer (2009). For the moment, we only consider the case of lists, and throughout, only parametrically polymorphic functions to bidirectionalize (no ad-hoc polymorphism). So we consider functions get $::[\alpha] \rightarrow[\alpha]$.

The intuition underlying the method of Voigtländer (2009) is that put can gain information about the get-function by applying it to suitable inputs. The key is that get is polymorphic over the element type $\alpha$. This entails that its behavior does not depend on any concrete list elements, but only on positional information, and this positional information can be observed explicitly by applying get to lists with fixed distinct elements. Particularly convenient are ascending lists over the natural numbers. Say get is tail, then every list $[1, \ldots, n]$ is mapped to $[2, \ldots, n]$, which allows put to see that the head element of the original source is absent from the view, hence cannot be affected by an update on the view, and hence should remain unchanged when propagating an updated view back into the source. And this observation can be transferred to other source lists than $[1, \ldots, n]$ just as well, even to lists over non-number types, thanks to parametric polymorphism (Reynolds 1983, Strachey 1967). Specifically, it is easy to derive from a "free theorem" (Wadler 1989) that for every get :: $[\alpha] \rightarrow[\alpha]$ and every list $s$, over arbitrary type, it holds that with $n=$ length $s$ and $t^{\prime} \equiv$ get $[1 \ldots n]$,

$$
\text { length }(\text { get } s)=\text { length } t^{\prime}
$$

as well as for every $1 \leqslant j \leqslant$ length (get $s$ ),

$$
(\text { get } s) ! !(j-1) \equiv s ! !\left(\left(t^{\prime} ! !(j-1)\right)-1\right) .
$$


(Note that !!, Haskell's operator for indexing into lists, starts counting from 0 , hence the occurrences of -1 .) Putting the above as a picture:

$$
\begin{gathered}
{[1, \ldots, n] \underset{\text { get }}{ } \underset{\text { vimplies }}{\longrightarrow}\left[t_{1}^{\prime}, \ldots, t_{m}^{\prime}\right]} \\
{\left[s_{1}, \ldots, s_{n}\right] \stackrel{\text { get }}{\Longrightarrow}\left[s_{t_{1}^{\prime}}, \ldots, s_{t_{m}^{\prime}}\right]}
\end{gathered}
$$

(where $\left[t_{1}^{\prime}, \ldots, t_{m}^{\prime}\right]$ could be the empty list, just as $[1 \ldots n] \equiv[1, \ldots, n]$ could be).

Let us further consider the tail example as in the middle of the previous paragraph. First, put should find out to what element in an original source $s$ each element in an updated view $v^{\prime}$ corresponds. Assume $s$ has length $n$. Then by applying tail to the same-length list $[1 \ldots n]$, put learns that the original view from which $v^{\prime}$ was obtained by updating had length $n-1$, and also to what element in $s$ each element in that original view corresponded. Being conservative, the original semantic bidirectionalization method will only accept $v^{\prime}$ if it has retained that length $n-1$. For then, we also know directly the associations between elements in $v^{\prime}$ and positions in the original source. Now, to produce the updated source, we can go over all positions in $[1 \ldots n]$ and fill each with the associated value from $v^{\prime}$. For positions for which there is no corresponding value in $v^{\prime}$, because these positions were omitted when applying tail to $[1 \ldots n]$, we can look up the correct value in $s$ rather than in $v^{\prime}$. For the concrete example, this will only concern position 1 , for which we naturally take over the head element from $s$ (also see the picture below).

Actually, "going over all positions in $[1 \ldots n]$ and filling each with the associated value from $v^{\prime}$ (or from $s$ if non-existent in $v^{\prime}$ )" can be a problematic task: what if two values in $v^{\prime}$ would associate with the same index position (as could easily happen if instead of tail we have a get-function that duplicates some of its list elements)? Ignoring that difficulty for the moment (but coming back to it soon, in Section 4.1, the above strategy works for general get. In short, given $s$, produce a "template" $t \equiv[1 \ldots n]$ of the same length, together with an association $g$ between natural numbers in that template and the corresponding values in $s$. Then apply get to $t$ and produce a further association $h$ by matching this template view $t^{\prime}$ with the updated proper value view $v^{\prime}$. Combine the two associations into a single one $h^{\prime}$, giving precedence to $h$ whenever a natural numbers template index is found in both $h$ and $g$ (or first reduce $g$ to $g^{\prime}$ by discarding all entries for index values that occur in get $[1 \ldots n]$; $h$ and $g^{\prime}$ will then have disjoint domains and together exactly cover $\{1, \ldots, n\}$ ). Finally, produce an updated source by filling all positions in $[1 \ldots n]$ with their associated values according to $h^{\prime}$. So for $s \equiv\left[s_{1}, \ldots, s_{n}\right]$ and $v^{\prime} \equiv\left[x_{1}, \ldots, x_{m}\right]$, set $p u t s v^{\prime} \equiv$ Just $\left[y_{1}, \ldots, y_{n}\right]$, where $y_{i} \equiv x_{j}$ if $i=t_{j}^{\prime}$ (with get $[1 \ldots n] \equiv\left[t_{1}^{\prime}, \ldots, t_{m}^{\prime}\right]$ ), and $y_{i} \equiv s_{i}$ otherwise. On the tail example:

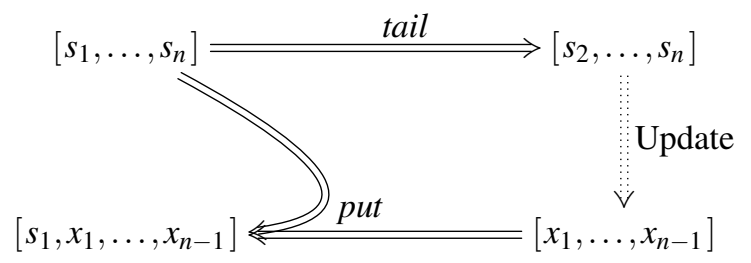


since tail $[1 \ldots n] \equiv[2 \ldots n]$ and hence $2=t_{1}^{\prime}, 3=t_{2}^{\prime}, \ldots, n=t_{n-1}^{\prime}$, and $1 \neq t_{j}^{\prime}$ for all $j$.

The above strategy (plus proper handling of get-functions that duplicate list elements) is what Voigtländer (2009) implements (for the special case get :: $[\alpha] \rightarrow[\alpha]$ ). We recall the corresponding Haskell definitions, reformulating just a bit by writing the higher-order bff-function ${ }^{1}$ that turns get into put in monadic style (Wadler 1992) to provide for more convenient error handling $\left.\right|^{2}$ The type class constraints $\mathrm{Eq}$ are for ensuring that list entries (of abstract type $\alpha$ ) can be compared for equality using $==$, as needed in assoc ${ }^{\beta}$

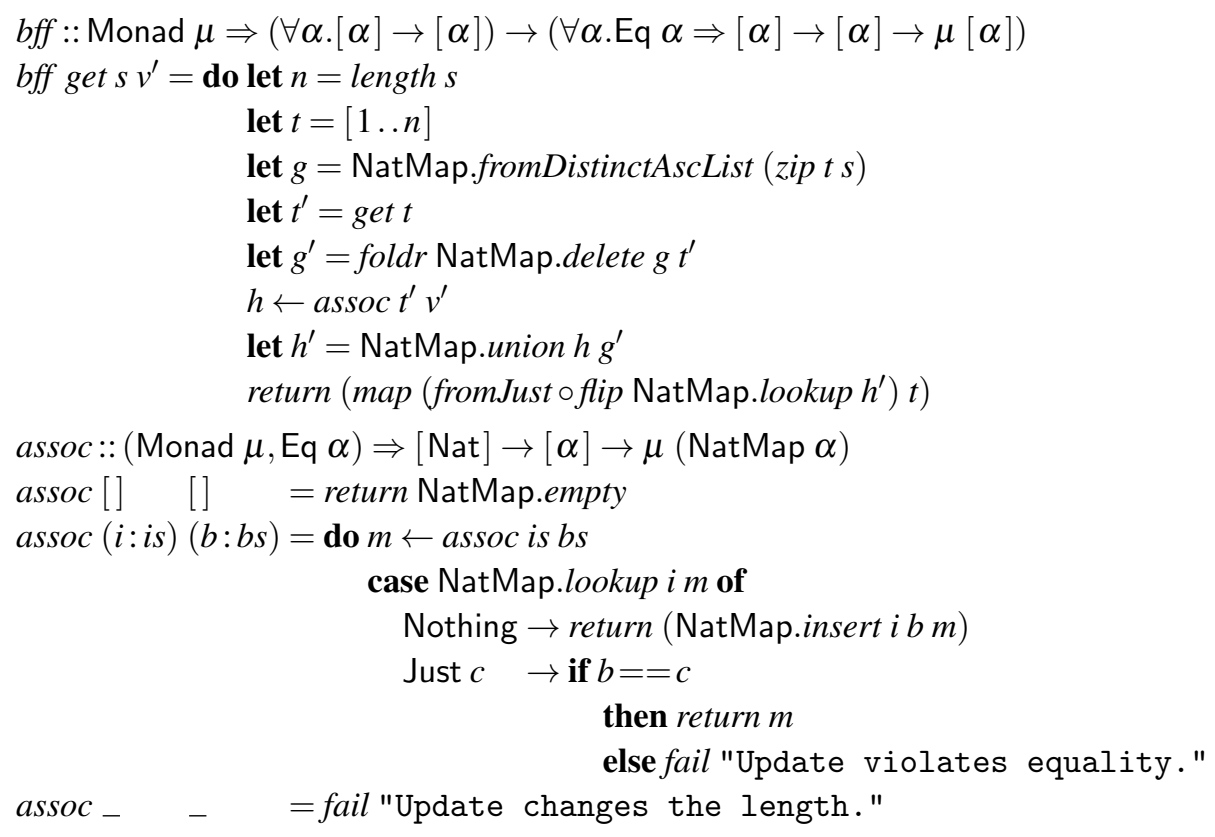

We use (here and later) some functions from an assumed module Data.NatMap. Their type signatures, which should provide sufficient documentation, are given as follows: ${ }^{4}$

$$
\begin{array}{ll}
\text { fromDistinctList } & ::[(\text { Nat }, \alpha)] \rightarrow \text { NatMap } \alpha \\
\text { fromDistinctAscList }::[(\text { Nat }, \alpha)] \rightarrow \text { NatMap } \alpha \\
\text { empty } & :: \text { NatMap } \alpha \\
\text { insert } & :: \text { Nat } \rightarrow \alpha \rightarrow \text { NatMap } \alpha \rightarrow \text { NatMap } \alpha \\
\text { delete } & :: \text { Nat } \rightarrow \text { NatMap } \alpha \rightarrow \text { NatMap } \alpha \\
\text { union } & :: \text { NatMap } \alpha \rightarrow \operatorname{NatMap} \alpha \rightarrow \text { NatMap } \alpha \\
\text { lookup } & :: \text { Nat } \rightarrow \text { NatMap } \alpha \rightarrow \text { Maybe } \alpha
\end{array}
$$

1 The name bff is an abbreviation of the paper title "Bidirectionalization for Free!".

2 We will only ever specialize $\mu$ to Maybe in the paper, but when running the code it is convenient to be able to (even silently) use an arbitrary monad. For example, just running examples in the interpreter can directly use the $\mathrm{IO}$ monad and thus give unwrapped outputs - unless there is a failure, of course. Monads in Haskell are not plain monads; they include a fail method. In the Maybe monad, we have fail $s=$ Nothing.

3 Note that $==$ is programmed equality, not in general semantic equivalence $\equiv$.

4 The function fromDistinctAscList can assume that its argument has Nat keys in ascending order, and thus can work more efficiently than plain fromDistinctList. 
Actually, Voigtländer (2009), and also Voigtländer et al. (2010), use slight variants (conceptually) of $b f f$ above where $g^{\prime} \equiv g$, i.e., the line let $g^{\prime}=$ fold $r$ NatMap.delete $g t^{\prime}$ is not present. The variation to use the reduced $g^{\prime}$ comes from Foster et al. (2012). This version is semantically equivalent to the earlier versions (since NatMap.union is assumed to be left-biased for natural numbers occurring as keys in both its input maps), but a difference appears when one starts to refactor $b f f$ to deal with view updates that change the shape. Then, in this paper from Section 4.4 onwards, the choice of $g^{\prime}$ vs. $g$ becomes relevant. We will return to this discussion there, after Theorem 6 .

The following theorem is essentially (up to the different way of expressing partiality of put $\equiv$ bff get) what is proved by Voigtländer (2009) in his Theorems 1 and 2, based on the key statements (3) and (4).

Theorem 1. Let get $::[\alpha] \rightarrow[\alpha]$ and let $\tau$ be a type that is an instance of the type class Eq in such a way that the definition given for $==$ makes it reflexive, symmetric, and transitive.

- For every $s::[\tau]$, bff get $s$ (get $s)::$ Maybe $[\tau] \equiv$ Just $s$.

- For every $s, v^{\prime}, s^{\prime}::[\tau]$, if bff get $s v^{\prime}::$ Maybe $[\tau] \equiv$ Just $s^{\prime}$, then get $s^{\prime}==v^{\prime}$.

Corollary 1. Let get $::[\alpha] \rightarrow[\alpha]$ and let $\tau$ be a type that is an instance of Eq in such a way that the definition given for $==$ agrees with $\equiv$. Then

$$
\text { bff get }::[\tau] \rightarrow[\tau] \rightarrow \text { Maybe }[\tau]
$$

is consistent (in the sense of Definition 1 for get $::[\tau] \rightarrow[\tau]$.

Applying semantic bidirectionalization is very easy. We simply call bff with the getfunction we want to bidirectionalize. The following two examples will also be used for later discussions.

Running Example 1. Assume our get-function is such that it sieves a list to keep only every second element, as exemplified with the following calls:

\begin{tabular}{c|c|c|c|c|c|c|c}
$s$ & "" & "a" & "ab" & "abc" & "abcd" & "abcde" & {$[1,2,3,4,5]$} \\
\hline get $_{1} s$ & " " & " " & "b" & "b" & "bd" & "bd" & {$[2,4]$}
\end{tabular}

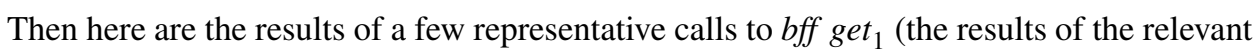
calls to get $_{1}$ are all the same):

\begin{tabular}{ccc|l}
$s$ & get $_{1} s$ & $v^{\prime}$ & bff get $_{1} s v^{\prime}$ \\
\hline "abcd" & "bd" & "x" & Nothing \\
"abcd" & "bd" & "xy" & Just "axcy" \\
"abcd" & "bd" & "xyz" & Nothing \\
"abcde" & "bd" & "x" & Nothing \\
"abcde" & "bd" & "xy" & Just "axcye" \\
"abcde" & "bd" & "xyz" & Nothing
\end{tabular}

We see (and indeed it holds in general) that bff get ${ }_{1} s v^{\prime}$ fails if and only if length $\left(\right.$ get $\left._{1} s\right) \neq$ length $v^{\prime}$. If it succeeds, it mixes the elements of $s$ and $v^{\prime}$ in an appropriate fashion. In a similar fashion as earlier for the tail example, see (5), the behavior here (specifically, the 
last but one line of the second table above) can be explained as follows:

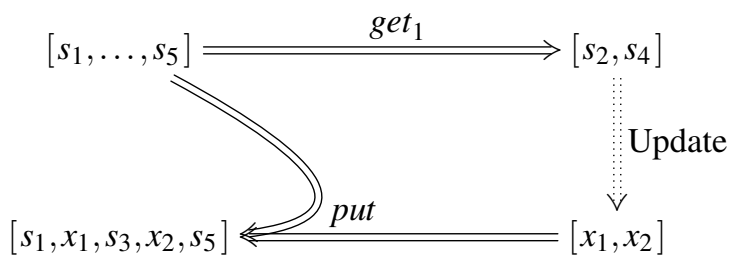

due to $\operatorname{get}_{1}[1 . .5] \equiv[2,4]$ and hence $2=t_{1}^{\prime}, 4=t_{2}^{\prime}$, and $1,3,5 \neq t_{j}^{\prime}$ for all $j$.

Running Example 2. Assume our get-function is such that it keeps every element of a list except for the last one, e.g.:

\begin{tabular}{c|c|c|c|c|c|c|c}
$s$ & "" & "a" & "ab" & "abc" & "abcd" & "abcde" & {$[1,2,3,4,5]$} \\
\hline get $_{2} s$ & "" & "" & "a" & "ab" & "abc" & "abcd" & {$[1,2,3,4]$}
\end{tabular}

Again, semantic bidirectionalization allows no view updates that change the shape, so bff get $t_{2} s v^{\prime}$ will only be successful if length $\left(\right.$ get $\left._{2} s\right)=$ length $v^{\prime}$, e.g.:

\begin{tabular}{ccc|l}
$s$ & get $_{2} s$ & $v^{\prime}$ & bff get g $_{2}$ \\
\hline$" "$ & $" 1$ & "" & Just "" \\
$" "$ & " & "x" & Nothing \\
"a" & $" "$ & $" "$ & Just "a" \\
"a" & "" & "x" & Nothing \\
"ab" & "a" & "" & Nothing \\
"ab" & "a" & "x" & Just "xb" \\
"ab" & "a" & "xy" & Nothing \\
"abc" & "ab" & "x" & Nothing \\
"abc" & "ab" & "xy" & Just "xyc"
\end{tabular}

\section{Refactoring Semantic Bidirectionalization to Enable "Plug-ins"}

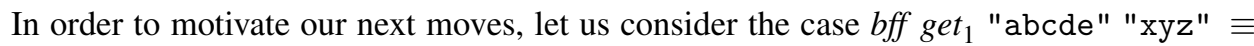
Nothing from Example 1 in the previous section. What makes

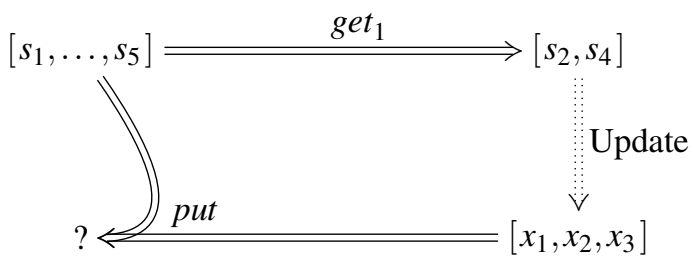

fail, in contrast to $(6)$, is that we cannot simply (as there) set put $\left[s_{1}, \ldots, s_{5}\right]\left[x_{1}, x_{2}, x_{3}\right] \equiv$ Just $\left[y_{1}, \ldots, y_{5}\right]$ with the $y_{i}$ appropriately chosen from among the $s_{i}$ and $x_{j}$. After all, no such choice(s) will ever make get ${ }_{1}\left[y_{1}, \ldots, y_{5}\right] \equiv\left[x_{1}, x_{2}, x_{3}\right]$ true, as the second of our consistency conditions would demand (cf. Definition 11). The problem is that length $n=5$ on the input side does not fit length $m=3$ on the (updated) output side. If, however, from $n=5$ and $m=3$ we could deduce an appropriate choice of length for the updated input 
list, say $n^{\prime}=6$ (or alternatively, $n^{\prime}=7$ ), then we could set the desired $s^{\prime}$ to $\left[y_{1}, \ldots, y_{6}\right]$ with $y_{i} \equiv x_{j}$ if $i=t_{j}^{\prime}$, where $\operatorname{get}_{1}[1 \ldots 6] \equiv\left[t_{1}^{\prime}, t_{2}^{\prime}, t_{3}^{\prime}\right]$, and $y_{i} \equiv s_{i}$ otherwise ${ }^{5}$

Determining $n^{\prime}-$ given get, $n$, and $m-$ can be considered as a separate problem, which is not solved (or solvable) by the semantic bidirectionalization technique itself. The idea now, already of Voigtländer et al. (2010), is to outsource this separate problem. If we abstract from the concrete list elements, instead considering (in the case of the above example) the following problem on the shape level:

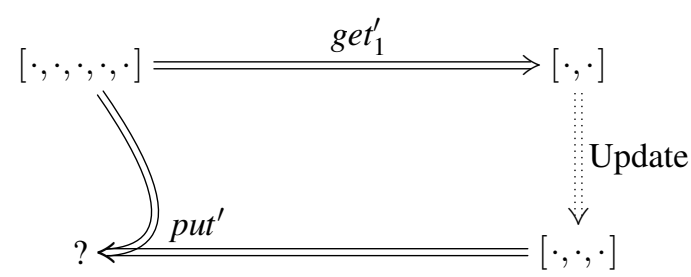

then maybe we are in better luck. Indeed, Voigtländer et al. (2010) showed that the syntactic bidirectionalization technique of Matsuda et al.(2007) can profit from such abstraction, and successfully generate put $^{\prime}$ (on the shape level) even in cases where it fails to generate (an as useful) put for the original problem. Next we show how to go about this separation of concerns in general, and to prepare integration of arbitrary shape bidirectionalizer plug-ins (that of Matsuda et al. or others). But beforehand, there is one more issue to consider.

The issue is that we have not yet discussed here, although of course we did so in the original work on semantic bidirectionalization (Voigtländer 2009, in detail at the end of Section 2 and start of Section 3), whether/how to deal with get-functions that duplicate input list elements. Here we shall shortly outlaw any duplication of list elements. Formally, we will consider only functions get $::[\alpha] \rightarrow[\alpha]$ such that for every $n::$ Nat, get $[1 \ldots n]$ contains no duplicates. We call such a function semantically affine. The property will clearly be fulfilled if get's syntactic definition is affine (i.e., if no variable occurs more than once in a single right-hand side), but it can also hold in other cases. In the next subsection we explain why we need this restriction; the reader less interested in the theoretical argument may want to skip that and go directly to Section 4.2 On the practical side, the restriction to semantically affine get-functions does not cost us all too much additionally in terms of reducing reach. In particular, the syntactic bidirectionalization technique of Matsuda et al. (2007), with which we combine semantic bidirectionalization in Section 5.3 as one chief result (Voigtländer et al. 2010) of our overall approach, is itself already unable to deal with syntactically non-affine functions.

\subsection{Semantic Bidirectionalization and Duplication of List Elements}

So how can semantic bidirectionalization deal with get-functions that duplicate input list elements? First of all, this issue is exactly what leads to the somewhat complicated definition of assoc in the previous section and to the references to Eq and $==$ in the function

5 If we were to, alternatively, choose $n^{\prime}=7$, then some default value would have to be brought into play, because for $i=7$ there is no $x_{j}$ with $7=t_{j}^{\prime}$, but also no $s_{7}$. 


\section{J. Voigtländer et al.}

definitions and in Theorem 1 and Corollary 1. More specifically, the need arises because we want to ensure that $b f f$ satisfies some form of the PutGet law (ultimately, expressed as the second of the two points stated in Theorem 1 . The problem for bff is to come up with an $s^{\prime}$ such that

$$
\text { length }\left(\text { get } s^{\prime}\right)=\text { length } v^{\prime}
$$

and for every $1 \leqslant j \leqslant$ length (get $\left.s^{\prime}\right)$, ideally

$$
\left(\text { get } s^{\prime}\right) ! !(j-1) \equiv v^{\prime} ! !(j-1),
$$

though we would actually be content with $==$ instead of $\equiv$. What do we have to go by for proving these two statements? Well, of course (3) and 4, applied to $s^{\prime}$. For length $\left(\right.$ get $\left.s^{\prime}\right)=$ length $v^{\prime}, 3$ already does half the job, leaving us with the proof obligation

$$
\text { length } t^{\prime}=\text { length } v^{\prime} \text {. }
$$

But this equality is ensured if the assoc-call on $t^{\prime}$ and $v^{\prime}$ inside $b f f$ succeeded (and only then do we have to prove anything about $s^{\prime}$ at all, cf. the exact formulation of the second point stated in Theorem 1). For $\left(\right.$ get $\left.s^{\prime}\right) ! !(j-1) \equiv v^{\prime} ! !(j-1)$, (4) looks quite useful, leaving us with the proof obligation

$$
s^{\prime} ! !\left(\left(t^{\prime} ! !(j-1)\right)-1\right) \equiv v^{\prime} ! !(j-1),
$$

where $n=$ length $s^{\prime}$ and $t^{\prime} \equiv$ get $[1 \ldots n]$. And indeed, the fact that when filling up $s^{\prime}$ in the last line of the definition of $b f f$, lookup for index positions that are elements of $t^{\prime}$ leads to lookup in $h$ - which was obtained through associating, by position, elements of $t^{\prime}$ and of $v^{\prime}$ - seems to indicate that we are fine. But, actually (8) is treacherous as a "definition" (implemented in $b f f$ ) for elements of $s^{\prime}$ at positions corresponding to elements of $t^{\prime}$. It is not actually well-defined in general! What if there are $j$ and $j^{\prime}$ such that $t^{\prime} ! !(j-1)=t^{\prime} ! !\left(j^{\prime}-1\right)$ ? Then (8) tries to "assign" two potentially different values $v^{\prime} ! !(j-1)$ and $v^{\prime} ! !\left(j^{\prime}-1\right)$ to the same position of $s^{\prime}$. The assoc-function as given in the previous section prevents this from happening, at the price of additionally performing equality checks (using $==$ ).

As a concrete example, consider a function get that maps every singleton list $s$ to $s+s$ (and all other lists to [], say). Let us look at a specific case $s=$ "a", and suppose the view "aa" is updated to "bc". Should we take this as suggesting a replacement of ' $a$ ' by ' $b$ ' or by 'c', i.e., do we want bff get "a" "bc" to be Just "b" or Just "c"? Neither makes sense, since neither get "b" nor get " $\mathrm{c}$ " is "bc". So the only possibility for bff is to return Nothing.

Restricting to semantically affine get-functions is not just the easiest way out of the complications described above. At a deeper level, it is really fundamental to a successful separate treatment of shapes as we have in mind. If the problem of coming up, for given $v^{\prime}$ (and $s$ ), with an $s^{\prime}$ such that get $s^{\prime}$ equals $v^{\prime}$ is to be decomposed in such a way that we first try to determine the length of $s^{\prime}$ from only the length of $v^{\prime}$ (and that of $s$ ), then we cannot afford to have to be concerned about the inner structure of get $[1 \ldots n]$ (or actually of get $\left[1 \ldots n^{\prime}\right]$ for some new $n^{\prime}$ potentially different from $n=$ length $s$ ) in order to eventually make sense of (8). Instead, we should only have to be concerned about the shape aspects, as in the other proof obligation (7). And indeed, if given the length of $v^{\prime}$ (and that of $s$ ) we manage to find an $n^{\prime}$ such that (7) holds for $t^{\prime} \equiv$ get $\left[1 \ldots n^{\prime}\right]$, then only under the 
semantic affineness assumption will we be guaranteed - no matter how $v^{\prime}$ looks internally - to be able to fill the list positions of $s^{\prime}$ (of length $n^{\prime}$ ) in such a way that get $s^{\prime}$ equals $v^{\prime}$. This is because (8) is not only sufficient, but actually necessary, and as soon as $t^{\prime}$ contains duplicates an adversary could come up with list elements for $v^{\prime}$ such that $s^{\prime}$ cannot exist (not even after replacing $\equiv$ by $==$ in (8); assuming the type of elements of $v^{\prime}$ has at least two non-equal values).

\subsection{Specializing Semantic Bidirectionalization to Semantically Affine get-Functions}

We define

$$
b f f_{\text {affine }}:: \text { Monad } \mu \Rightarrow(\forall \alpha .[\alpha] \rightarrow[\alpha]) \rightarrow(\forall \alpha .[\alpha] \rightarrow[\alpha] \rightarrow \mu[\alpha])
$$

like bff (but note the different type), except that the call to assoc is replaced by a call, with the same arguments, to the following function, not performing any equality checks:

$$
\begin{aligned}
& \operatorname{assoc}^{\prime}:: \text { Monad } \mu \Rightarrow[\text { Nat }] \rightarrow[\alpha] \rightarrow \mu(\text { NatMap } \alpha) \\
& \text { assoc }^{\prime}[] \quad[] \quad=\text { return NatMap.empty } \\
& \operatorname{assoc}^{\prime}(i: i s)(b: b s)=\mathbf{d o} m \leftarrow a s s o c^{\prime} \text { is } b s \\
& \text { return (NatMap.insert i } b \text { m) } \\
& \text { assoc }^{\prime}{ }_{\text {_ _ _ _ }} \quad \text { fail "Update changes the length." }
\end{aligned}
$$

The proof of the following theorem is very similar to that of Theorem 11 additionally using semantic affineness of get in a straightforward way.

Theorem 2. Let get $::[\alpha] \rightarrow[\alpha]$ be semantically affine. For every type $\tau$,

$$
b f f_{\text {affine }} \text { get }::[\tau] \rightarrow[\tau] \rightarrow \text { Maybe }[\tau]
$$

is consistent for get $::[\tau] \rightarrow[\tau]$.

But semantic affineness gives us more. It rules out one important cause (namely potential equality mismatch in $v^{\prime}$ ) for a potential failure of view update. As a consequence, we can now formulate a sufficient condition for a successful update.

Definition 2. We say that a function put :: $[\tau] \rightarrow[\tau] \rightarrow$ Maybe $[\tau]$ (for some type $\tau$ ) is fixed-shape-friendly for get $::[\tau] \rightarrow[\tau]$ if for every $s, v^{\prime}::[\tau]$, if length $($ get $s)=$ length $v^{\prime}$, then put $s v^{\prime} \equiv$ Just $s^{\prime}$ for some $s^{\prime}::[\tau]$.

Note that the original bff get $::[\tau] \rightarrow[\tau] \rightarrow$ Maybe $[\tau]$ from Section 3 is not in general fixed-shape-friendly for get-functions that are not semantically affine. On the other hand, bff $f_{\text {affine }} g e t::[\tau] \rightarrow[\tau] \rightarrow$ Maybe $[\tau]$ is not even generally consistent for get-functions that are not semantically affine. But when we $d o$ restrict get-functions to be semantically affine, we have consistency by the above theorem, and can moreover prove the following one.

Theorem 3. Let get :: $[\alpha] \rightarrow[\alpha]$ be semantically affine. For every type $\tau$,

$$
b f f_{\text {affine }} \text { get }::[\tau] \rightarrow[\tau] \rightarrow \text { Maybe }[\tau]
$$

is fixed-shape-friendly for get $::[\tau] \rightarrow[\tau]$. 
For the proof, we basically just observe that the last defining equation of $a s s o c^{\prime}$ will never be reached if the argument lists are of the same length.

We can also give a negative statement about updateability (which also holds for the bff from Section 3 , of course).

Theorem 4. Let get $::[\alpha] \rightarrow[\alpha]$. For every type $\tau$ and $s, v^{\prime}::[\tau]$, if length $($ get $s) \neq$ length $v^{\prime}$, then bff affine get $s v^{\prime}::$ Maybe $[\tau] \equiv$ Nothing.

For the proof, we observe that the last defining equation of $a s s o c^{\prime}$ (or assoc) is reached if the argument lists are of different lengths.

\subsection{Decomposing to Expose the Shape Aspect}

We refactor $b f f_{\text {affine }}$ to make the treatment of shapes (list lengths) more explicit. To that end, we first define a function $s p u t_{\text {naive }}$, depending on get $::[\alpha] \rightarrow[\alpha]$, as follows:

$$
\begin{aligned}
\text { sput }_{\text {naive }}:: \text { Monad } \mu \Rightarrow & (\forall \alpha \cdot[\alpha] \rightarrow[\alpha]) \rightarrow(\text { Nat } \rightarrow \text { Nat } \rightarrow \mu \text { Nat }) \\
\text { sput }_{\text {naive }} \text { get } l_{s} l_{v^{\prime}}= & \text { if } \text { length }\left(\text { get }\left[1 \ldots l_{s}\right]\right)==l_{v^{\prime}} \\
& \text { then } \text { return } l_{s} \\
& \text { else } \text { fail } \text { "Update changes the length." }
\end{aligned}
$$

Using that function, we then define $b f f_{\text {refac }}$ as follows:

$$
\begin{aligned}
b f f_{\text {refac }}:: \text { Monad } \mu \Rightarrow & (\forall \alpha .[\alpha] \rightarrow[\alpha]) \rightarrow(\forall \alpha .[\alpha] \rightarrow[\alpha] \rightarrow \mu[\alpha]) \\
\text { bff } f_{\text {refac }} \text { get } s v^{\prime}=\text { do let } n=\text { length } s & \text { let } t=[1 \ldots n] \\
& \text { let } g=\text { NatMap.fromDistinctAscList }(\text { zip t } s) \\
& \text { let } g^{\prime}=\text { foldr NatMap.delete } g(\text { get } t) \\
& n^{\prime} \leftarrow \text { sput } \text { naive }_{\text {net } n\left(\text { length } v^{\prime}\right)} \\
& \text { let } t=\left[1 \ldots n^{\prime}\right] \\
& \text { let } h=\text { NatMap.fromDistinctList }\left(\text { zip }(\text { get } t) v^{\prime}\right) \\
& \text { let } h^{\prime}=\text { NatMap.union } h g^{\prime} \\
& \text { return }\left(\text { map }\left(\text { fromJust } \circ \text { flip NatMap.lookup } h^{\prime}\right) t\right)
\end{aligned}
$$

The refactoring consists of:

- making the check for equal length of get $[1$.. (length $s)]$ and $v^{\prime}$, otherwise performed inside $a s s o c^{\prime}$, explicit, and outsourcing it to $s p u t_{\text {naive }}$, and

- realizing that once this check was successful, the role of $a s s o c^{\prime}$ can be taken over by zip and NatMap.fromDistinctList.

Note that the second local binding for $t$ inside $b f f f_{\text {refac }}$ shadows the earlier one, but that actually the two values bound will be identical here, since due to the behavior of $s p u t_{\text {naive }}$, if the second binding is reached at all, then $n^{\prime}$ will be identical to $n$. (That will change in the next subsection.)

The following lemma establishes that the above refactoring is indeed correct, and thus transports the (good and bad) properties of $b f f_{\text {affine }}$ to $b f f_{\text {refac }}$.

Lemma 1. Let get $::[\alpha] \rightarrow[\alpha]$. For every type $\tau$ and $s, v^{\prime}::[\tau]$, we have

$$
b f f_{\text {affine }} \text { get } s v^{\prime}:: \text { Maybe }[\tau] \equiv b f f_{\text {refac }} \text { get } s v^{\prime}:: \text { Maybe }[\tau] \text {. }
$$


Corollary 2. Let get :: $[\alpha] \rightarrow[\alpha]$ be semantically affine. For every type $\tau$,

$$
\text { bff refac } g e t::[\tau] \rightarrow[\tau] \rightarrow \text { Maybe }[\tau]
$$

is consistent for get $::[\tau] \rightarrow[\tau]$.

Corollary 3. Let get :: $[\alpha] \rightarrow[\alpha]$ be semantically affine. For every type $\tau$,

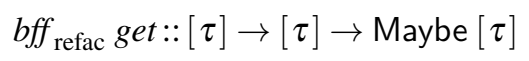

is fixed-shape-friendly for get $::[\tau] \rightarrow[\tau]$.

Corollary 4. Let get :: $[\alpha] \rightarrow[\alpha]$. For every type $\tau$ and $s, v^{\prime}::[\tau]$, if length (get $\left.s\right) \neq$ length $v^{\prime}$, then bff refac $g e t s v^{\prime}:$ : Maybe $[\tau] \equiv$ Nothing.

The motivation for our refactoring above is that we make explicit, in $s p u t_{\text {naive }}$, what happens on the shape level, namely that only updated views with the same length as the original view can be accepted, and that the length of the source will never be changed. By "playing" with sput $t_{\text {naive }}$, or rather replacing it, we can change that behavior.

\subsection{Enabling "Plug-ins"}

The key idea in the previous subsection is abstraction: from lists to list lengths (generally, from data structures to their shapes). We can define a function shapify as follows:

$$
\begin{aligned}
& \text { shapify }::(\forall \alpha .[\alpha] \rightarrow[\alpha]) \rightarrow(\mathrm{Nat} \rightarrow \mathrm{Nat}) \\
& \text { shapify get } n=\text { length }(\text { get }[1 \ldots n])
\end{aligned}
$$

Actually, one can often directly derive, from get, a simple syntactic definition for a function sget semantically equivalent to shapify get. That will be an important point in Section 5.3 . But for the moment, we simply take the above definition.

Next, we assume that some function sput is given, with the following type:

$$
\text { sput }:: \mathrm{Nat} \rightarrow \mathrm{Nat} \rightarrow \text { Maybe Nat, }
$$

and that sput is consistent for shapify get. Of course, sput $\equiv s p u t_{\text {naive }} g e t$ is always a valid choice, but for many get-functions there will be better alternatives!

We now define $b f f_{\text {plug }}$ as below. There are three differences from $b f f_{\text {refac }}$ : instead of calling out to $s p u t_{\text {naive }}$ get, we call out to an (additional, besides get) function argument sput (that is again itself a function), we generate an error message in case that sput fails (previously this was done directly in $s p u t_{\text {naive }}$ ), and we drop the fromJust from the last (return-) line. The latter change introduces an extra Maybe type constructor in the output list type, and is done to deal with list positions for which no data is known, neither from the original source nor from the updated view.

$$
\begin{aligned}
& b f f_{\text {plug }}:: \text { Monad } \mu \Rightarrow(\forall \alpha .[\alpha] \rightarrow[\alpha]) \rightarrow(\text { Nat } \rightarrow \text { Nat } \rightarrow \text { Maybe Nat }) \\
& \rightarrow(\forall \alpha \cdot[\alpha] \rightarrow[\alpha] \rightarrow \mu[\text { Maybe } \alpha]) \\
& \text { bff plug get sput } s v^{\prime}=\text { do let } n=\text { length } s \\
& \text { let } t=[1 \ldots n] \\
&\text { let } g=\text { NatMap.fromDistinctAscList (zip t } s) \\
& \text { let } g^{\prime}=\text { foldr NatMap.delete } g(\text { get } t)
\end{aligned}
$$




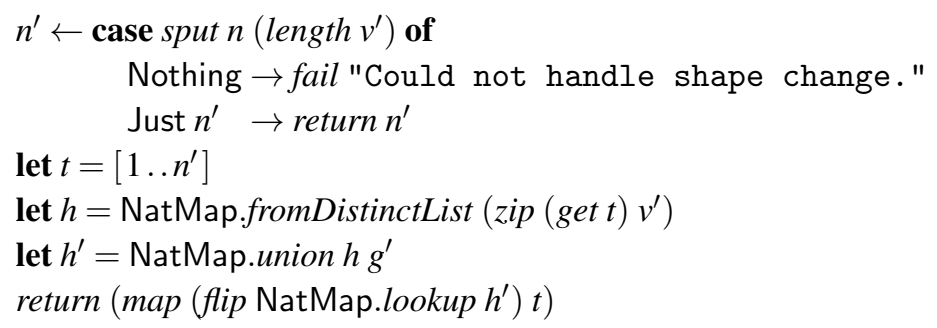

Note that now the second local binding for $t$, shadowing the first one, can really yield a different list, because it is no longer a given that $n^{\prime}$ is identical to $n$. Also at this point, it becomes relevant that we assume NatMap.union to be left-biased for natural numbers occurring as keys in both its input maps. That is important to guarantee precedence of $h$ over $g^{\prime}$ for positions of the output list that are represented both in the domain of $h$ (comprising all natural numbers that occur in get $\left[1 \ldots n^{\prime}\right]$ ) and in that of $g^{\prime}$ (comprising all natural numbers that occur in $[1 \ldots n]$ but not in get $[1 \ldots n])$.

The proof of the following theorem is then very similar to that of Theorem 11, but of course additionally uses the assumption about the relationship between sput and shapify get.

Theorem 5. Let get $::[\alpha] \rightarrow[\alpha]$ be semantically affine. Let sput be consistent for shapify get. Let $\tau$ be a type.

- For every $s::[\tau]$, bff $_{\text {plug }}$ get sput $s$ (get $\left.s\right)::$ Maybe [Maybe $\left.\tau\right] \equiv$ Just (map Just $s$ ).

- For every $s, v^{\prime}::[\tau]$ and $s^{\prime}::[$ Maybe $\tau]$, if

$$
\text { bff plug get sput } \left.s v^{\prime}:: \text { Maybe [Maybe } \tau\right] \equiv \text { Just } s^{\prime},
$$

then get $s^{\prime} \equiv$ map Just $v^{\prime}$.

The following theorem can also be shown to hold.

Theorem 6. Let get $::[\alpha] \rightarrow[\alpha]$ be semantically affine. Let sput be consistent for shapify get. For every type $\tau$ and $s, v^{\prime}::[\tau]$, if length (get $\left.s\right)=$ length $v^{\prime}$, then bff plug get sput $s v^{\prime}::$ Maybe $[$ Maybe $\tau] \equiv$ Just (map Just $\left.s^{\prime}\right)$ for some $s^{\prime}::[\tau]$.

The proof is basically by observing that if we have length (get $s)=$ length $v^{\prime}$, then also shapify get (length $s)=$ length $v^{\prime}$, and thus, by consistency of sput for shapify get, inside the $b f f_{\text {plug }}$-definition $n^{\prime}$ will be successfully assigned the value $n$, and subsequently every index position from $t$ will lead to a successful lookup in $h^{\prime}$, because $h$ covers all such positions that also occur in get $t$ while $g^{\prime}$ covers exactly all the rest.

Neither Theorem 5 nor Theorem 6 says anything about when a Nothing can become manifest for the "inner" Maybe in the result type of bff plug get sput s $v^{\prime}$. This is so because such Nothing-values can only appear on the updated source side, and only if the shape was changed. We already mentioned earlier that $b f f_{\text {plug }}$ uses the extra Maybe type constructor to deal with positions in the output list for which no data is known, neither from the original source nor from the updated view. Let us discuss this in a bit more detail now, also returning to the discussion of the "choice of $g^{\prime}$ vs. $g$ " as promised directly after introducing bff in Section 3 (The reader less interested in the subtleties may want to skip over directly to the paragraph before Corollary 5) 
So what happens with $b f f_{\text {plug }}$ get sput $s v^{\prime}$ if $v^{\prime}$ does not have the same length as get $s$ ? Then $n^{\prime}$ will be different from $n$ in $b f f_{\text {plug }}$, and when going through the index positions from $t \equiv\left[1 \ldots n^{\prime}\right]$ to create $s^{\prime}$, some positions might not be found in the domain of $h^{\prime}$. Obviously (since the domain of $h^{\prime}$ is the union of the domains of $h$ and $g^{\prime}$ ), this will happen exactly for all natural numbers from "set" $S^{\prime}=\left[1 \ldots n^{\prime}\right]$ that occur neither in $V^{\prime}=$ get $\left[1 \ldots n^{\prime}\right]$ (the domain of $h$ ) nor in, with $S=[1 \ldots n]$ and $V=$ get $[1 \ldots n], S \backslash \backslash V$ (the domain of $g^{\prime}$, computed using the "set difference" operator $\backslash \backslash)$. We may picture the setup/connections here as follows (though we will never have reason to actually compute put $[1 \ldots n]\left(\right.$ get $\left.\left.\left[1 \ldots n^{\prime}\right]\right)\right)$ :

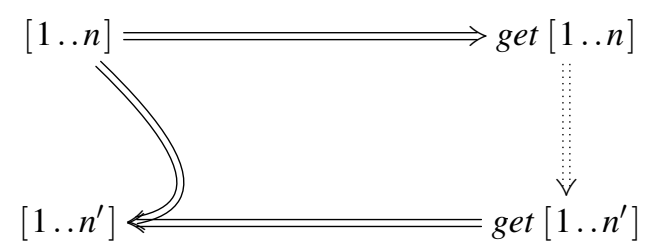

So, following the above observations about the domains of $h^{\prime}, h$, and $g^{\prime}$, all positions of $s^{\prime}$ that correspond to a natural number from

$$
X=\mathrm{S}^{\prime} \backslash \backslash\left(\mathrm{V}^{\prime} \cup(\mathrm{S} \backslash \backslash \mathrm{V})\right)
$$

will be filled with Nothing. Justifiedly so? Well, first of all, it is ensured that this will not happen for positions that correspond, after performing get, to elements/positions of $v^{\prime}$ Moreover, for various of the elements of $X$, namely for all elements of

$$
Y=\mathrm{S}^{\prime} \backslash \backslash\left(\mathrm{V}^{\prime} \cup \mathrm{S}\right)
$$

it is hardly conceivable what Just-value to provide for them. After all, $Y$ will only be nonempty if $n^{\prime}>n$, i.e., the update on the view (shape) triggered an update on the source that forced it to become longer, and any element of $Y$ will be a position for which we have no meaningful way to pick an element from the original source (precisely because it will be a position beyond the length $n$ of the original source) and for which we also cannot justify picking an element from the updated view (because it will be a position not occurring in $\mathrm{V}^{\prime}$, hence not corresponding to an element of the view list).

But one doubt may remain: what about elements of $V \cap S^{\prime} \backslash \backslash V^{\prime}$ ? This set is obtained as the difference $X \backslash Y$ (taking into account that $S \supseteq V$ ). So the elements in question are exactly the positions for which — since they appear in $X$ - we assign Nothing in $s^{\prime}$ even though they do not appear in $Y$. For them, it might seem tempting to lookup a value in $g$, which would correspond to accessing a position of the original source $s$ (which after all has length $n$, so we are guaranteed to find some value). Indeed, that is exactly what we did previously (Voigtländer et al. 2010, by not having the line let $g^{\prime}=$ fold $r$ NatMap.delete $g t^{\prime}$ in $b f f$ and $b f f_{\text {plug }}$, instead using $g^{\prime} \equiv g$ ). But morally it is actually wrong: we would fill a position in the updated source for which we have no support from the updated view, with a value from the original view. This seems too arbitrary. After all, the get-function

6 This, which is guaranteed by $\mathrm{V}^{\prime}$ being covered by $h$, is reflected in the second point stated in Theorem 5. which implies that all elements of get $s^{\prime}$ are Just-values. Of course, that point does not prevent other positions of $s^{\prime}$ from containing Nothing-values. 
will have a "reason" for omitting that position when going from a source of length $n^{\prime}$ to a corresponding view. If at all attempting to fill the position with an element from the situation before any update happens, we should attempt to explain it from the original source, not from the original view. If we are unable to do so, we are better off returning Nothing. We will again consider this issue, based on a concrete example - materializing the difference in this respect between what we did previously and what we do in this paper - towards the end of Section 5.1

Instead of producing Just- and Nothing-values, it is usually more convenient to simply use a default value for positions in the output list for which no data is known, neither from the original source nor from the updated view. Hence, we define a function $d b f f$ as follows:

$$
\begin{aligned}
\text { dbff }:: \text { Monad } \mu \Rightarrow & (\forall \alpha .[\alpha] \rightarrow[\alpha]) \rightarrow(\text { Nat } \rightarrow \text { Nat } \rightarrow \text { Maybe Nat }) \\
& \rightarrow(\forall \alpha . \alpha \rightarrow[\alpha] \rightarrow[\alpha] \rightarrow \mu[\alpha]) \\
\text { dbff get sput } d s v^{\prime}= & \text { do } s^{\prime} \leftarrow b f f \text { plug } \text { get sput s } v^{\prime} \\
& \quad \text { return }\left(\text { map }(\lambda \text { case }\{\text { Nothing } \rightarrow d ; \text { Just } y \rightarrow y\}) s^{\prime}\right)
\end{aligned}
$$

The following two statements are then relatively direct consequences of Theorems 5 and 6

Corollary 5. Let get:: $[\alpha] \rightarrow[\alpha]$ be semantically affine. Let sput be consistent for shapify get. For every type $\tau$ and $d:: \tau$,

$$
\text { dbff get sput } d::[\tau] \rightarrow[\tau] \rightarrow \text { Maybe }[\tau]
$$

is consistent for get $::[\tau] \rightarrow[\tau]$.

Corollary 6. Let get:: $[\alpha] \rightarrow[\alpha]$ be semantically affine. Let sput be consistent for shapify get. For every type $\tau$ and $d:: \tau$,

$$
\text { dbff get sput } d::[\tau] \rightarrow[\tau] \rightarrow \text { Maybe }[\tau]
$$

is fixed-shape-friendly for get $::[\tau] \rightarrow[\tau]$. (Moreover, the default value $d$ is not actually used in dbff get sput $d s v^{\prime}$ if length (get s) = length $v^{\prime}$.)

It is important to note that no general negative statement like Theorem 4 or Corollary 4 holds for $d b f f$ (or for $b f f_{\text {plug }}$ ). It all depends on the argument sput! If we find a good sput that is consistent for shapify get, then dbff get sput will also be good for get. This is where we can now plug in arbitrary "shape bidirectionalizers".

\section{Some Concrete "Plug-ins"}

\subsection{Manual Shape-Bidirectionalization}

In principle, a reasonable stance to take is that the programmer, who has programmed get, should also provide sput. After all, the programmer can be often expected to have a very good idea of how shape-changing updates should be dealt with.

Running Example 1 (continued, with manual provision of $s p u t$ ). Recall that get $_{1}$ sieves a list to keep only every second element. On the shape level this means to halve the length of the list, so an intuitive backwards transformation seems to be to double the length of any provided updated view list: 


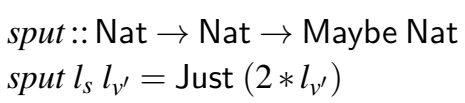

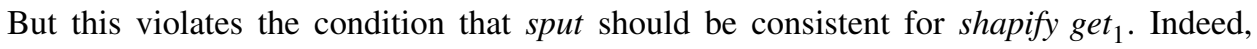
sput $l_{s}\left(\right.$ shapify get $\left.l_{1} l_{s}\right) \equiv$ Just $l_{s}$ does not hold for any odd natural number $l_{s}$. After all, shapify get $t_{1}$ is not exact halving, but actually halving with truncation. A natural remedy is to refine sput as follows:

$$
\begin{aligned}
& \text { sput }:: \text { Nat } \rightarrow \text { Nat } \rightarrow \text { Maybe Nat } \\
& \text { sput } l_{s} l_{v^{\prime}}=\text { Just }\left(2 * l_{v^{\prime}}+l_{s}{ }^{\prime} \bmod ^{\prime} 2\right)
\end{aligned}
$$

Then sput is consistent for shapify get ${ }_{1}$, and can thus be used as follows, with the guarantees

\begin{tabular}{|c|c|c|c|}
\hline$s$ & $\operatorname{get}_{1} s$ & $v^{\prime}$ & ${\text { dbff } \text { get }_{1} \text { sput }},{ }^{\prime} s v^{\prime}$ \\
\hline "abcd" & "bd" & "x" & Just "ax" \\
\hline "abcd" & "bd" & "xy" & Just "axcy" \\
\hline "abcd" & "bd" & "xyz" & Just "axcy z" \\
\hline "abcd" & "bd" & "xyzv" & Just "axcy z v" \\
\hline "abcde" & "bd" & "x" & Just "axc" \\
\hline "abcde" & "bd" & "xy" & Just "axcye" \\
\hline "abcde" & "bd" & "xyz" & Just "axcyez" \\
\hline "abcde" & "bd" & "xyzv" & Just "axcyez v " \\
\hline
\end{tabular}
from Corollaries 5 and 6 .

Note that when length $\left(\right.$ get $\left._{1} s\right) \neq$ length $v^{\prime}$, dbff get sput $^{\prime}, ' s v^{\prime}$ extends - making use of the default value - or shrinks the source list by a number of elements that is a multiple of two. All updates can now be successfully handled, much in contrast to earlier, when we used bff get $t_{1}$ instead of dbff $_{\text {get }}$ sput ' '. The moderate price to pay is that the programmer has to come up with sput.

Running Example 2 (continued, with manual provision of $s p u t$ ). Recall that get $_{2}$ keeps every element of a list except for the last one, and maps the empty list to itself. On the shape level this means that shapify get ${ }_{2}$ maps 0 to 0 , and every positive number to its predecessor. One possible choice for a consistent sput is thus:

$$
\begin{aligned}
& \text { sput }:: \text { Nat } \rightarrow \text { Nat } \rightarrow \text { Maybe Nat } \\
& \text { sput } 00 \\
& \begin{aligned}
& \text { sput } l_{s} l_{v^{\prime}} \mid l_{s}>0 \vee l_{v^{\prime}}>0=\text { Just } 0 \\
& \text { Just }\left(l_{v^{\prime}}+1\right)
\end{aligned}
\end{aligned}
$$


With it, we get:

\begin{tabular}{|c|c|c|c|}
\hline$S$ & get $_{2} s$ & $v^{\prime}$ & $d b f f g_{2} t_{2}$ spt ${ }^{\prime}, s v^{\prime}$ \\
\hline "1" & "1" & "1" & Just " " \\
\hline " " & " " & "x" & Just "x " \\
\hline " " & " " & "xy" & Just "xy " \\
\hline "a" & " " & " " & Just "a" \\
\hline "a" & " " & "x" & Just "x " \\
\hline "ab" & "a" & " " & Just " " \\
\hline "ab" & "a" & "x" & Just "xb" \\
\hline "ab" & "a" & "xy" & Just "xy " \\
\hline "abc" & "ab" & " " & Just " " \\
\hline "abc" & "ab" & "x" & Just "x " \\
\hline "abc" & "ab" & "xy" & Just "xyc" \\
\hline "abc" & "ab" & "xyz" & Just "xyz " \\
\hline
\end{tabular}

This is better than what we saw for this example in Section 3 , but still not perfect, since at some places the default value gets used where intuitively a specific value from the source list (namely the last element of $s$ ) would be appropriate instead. We will return to this aspect in Section 6

A related issue is that in the above table we see a manifest effect of the "choice of $g^{\prime}$ vs. $g$ " issue that was already mentioned after introducing bff in Section 3 and that was discussed after Theorem 6 in Section 4.4. Had we concerning that choice proceeded as

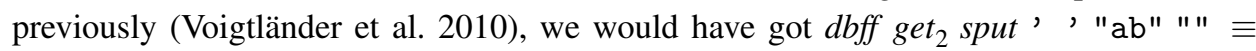

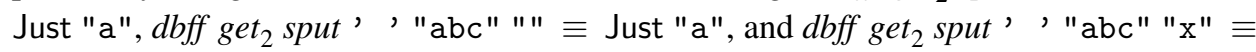
Just " $\mathrm{xb}$ ", but those ' $\mathrm{a}$ ' and ' $\mathrm{b}$ ' have no business appearing in the updated sources, since they are not (even) the last elements of the respective original sources.

It is worth pointing out that when writing sput, the programmer may well profit from a structured/combinator-based approach such as generic point-free lenses (Pacheco and Cunha 2010). Where writing get using the point-free combinators is hard (due to having to worry about the projection of elements and the inventing of them in the backwards direction), writing sget (and thus, due to the lens framework, immediately also sput) using the combinators could be much simpler.

\subsection{Shape-Bidirectionalization by Search}

Another viable option is to discover appropriate new source shapes by search. Specifically,

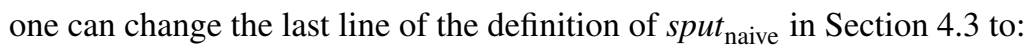

$$
\text { else return (head } \left.\left[l_{s^{\prime}} \mid l_{s^{\prime}} \leftarrow[0 . .] \text {, length }\left(\text { get }\left[1 \ldots l_{s^{\prime}}\right]\right)==l_{v^{\prime}}\right]\right)
$$

(which may of course lead to non-termination that is unavoidable in general) and use that version to obtain (partial function) sput from get. Actually, thanks to semantic affineness of get, it is sufficient to start the search for $l_{s^{\prime}}$ (after $l_{s}$ itself has been ruled ou 7 ) at $l_{v^{\prime}}$, i.e.,

7 We always need to check $l_{s}$ first, to guarantee the first of our consistency conditions (cf. Definition 1 . 
one can replace $[0 .$.$] by \left[l_{v^{\prime}} ..\right]$ above, or equivalently have altogether (and specialized to the Maybe monad, but nevertheless still returning a non-total sput in general):

$$
\begin{gathered}
\operatorname{sput}_{\text {search }}::(\forall \alpha .[\alpha] \rightarrow[\alpha]) \rightarrow(\text { Nat } \rightarrow \text { Nat } \rightarrow \text { Maybe Nat }) \\
\text { sput }_{\text {search }} \text { get } l_{s} l_{v^{\prime}}=\text { Just }\left(\text { head } \left[l_{s^{\prime}} \mid l_{s^{\prime}} \leftarrow l_{s}:\left(\left[l_{v^{\prime}} . .\right] \backslash \backslash\left[l_{s}\right]\right),\right.\right. \\
\text { length } \left.\left.\left(\text { get }\left[1 \ldots l_{s^{\prime}}\right]\right)==l_{v^{\prime}}\right]\right)
\end{gathered}
$$

One could even give the user some control over the "perfect updateability" achieved using pure search, enabling them to provide guidance via heuristics expressed as reorderings of the candidate list $\left[l_{v^{\prime}} ..\right]$. Here, we instead only consider the most basic search approach, on our two running examples.

Running Example 1 (with search instead of manual provision of $s p u t$ ). Applying $s p u t_{\text {search }}$ to get $_{1}$ yields a function semantically equivalent to the following one:

$$
\begin{aligned}
& \text { sput }:: \text { Nat } \rightarrow \text { Nat } \rightarrow \text { Maybe Nat } \\
& \text { sput } l_{s} l_{v^{\prime}}=\text { if } l_{s}{ }^{\circ} d i v^{\circ} 2==l_{v^{\prime}} \text { then Just } l_{s} \text { else Just }\left(2 * l_{v^{\prime}}\right)
\end{aligned}
$$

It is (by construction) consistent for shapify get $t_{1}$, and behaves like the second sput given for this example in Section 5.1. except when $l_{s}$ is odd and $l_{v^{\prime}}$ is not its (truncated) half. Concretely, this implies that (only) the following lines change compared to the corresponding input/output table from Section 5.1

\begin{tabular}{ccc|l}
$s$ & get $_{1} s$ & $v^{\prime}$ & dbff get $_{1}\left(\right.$ sput $_{\text {search }}$ get $\left._{1}\right){ }^{\prime}, s v^{\prime}$ \\
\hline "abcde" & "bd" & "x" & Just "ax" \\
"abcde" & "bd" & "xyz" & Just "axcyez" \\
"abcde" & "bd" & "xyzv" & Just "axcyez v"
\end{tabular}

Running Example 2 (with search instead of manual provision of $s p u t$ ). Applying $s p u t_{\text {search }}$ to get $_{2}$ yields a function semantically equivalent to the following one:

$$
\begin{aligned}
& \text { sput }:: \text { Nat } \rightarrow \text { Nat } \rightarrow \text { Maybe Nat } \\
& \text { sput } l_{s} 0 \mid l_{s} \leqslant 1=\text { Just } l_{s} \\
& \text { sput } l_{s} 0 \mid l_{s}>1=\text { Just } 0 \\
& \text { sput_ } l_{v^{\prime}} \mid l_{v^{\prime}}>0=\text { Just }\left(l_{v^{\prime}}+1\right)
\end{aligned}
$$

It differs from the one given for this example in Section 5.1 exactly when $l_{s}>1$ and $l_{v^{\prime}}=0$, so that we get changed behavior as follows:

$$
\begin{array}{ccc|l}
s & \text { get }_{2} s & v^{\prime} & \text { dbff get }_{2}\left(\text { sput }_{\text {search }} \text { get }_{2}\right),{ }^{\prime} s v^{\prime} \\
\hline \text { "ab" } & \text { "a" } & \text { "" } & \text { Just "" } \\
\text { "abc" } & \text { "ab" } & \text { "" } & \text { Just " " }
\end{array}
$$

\subsection{Combining Syntactic and Semantic Bidirectionalization}

The search approach from the previous subsection is attractive in its simplicity, and works reasonably well for our two running examples, but it is certainly not a panacea. Besides possible concerns about its efficiency in finding solutions, there is the problem that even if there is no solution (appropriate source shape for a given update configuration) at all, that fact will not be discovered (by leading to return value Nothing) in finite time. Also, 
formally legitimate updates found by search may be less meaningful to the user than ones obtained from more "intelligent" or "intuition-guided" shape bidirectionalizers. One possibility of the latter kind is to employ an existing bidirectionalization approach (Matsuda et al. 2007) based on constant complements (Bancilhon and Spyratos 1981).

The basic idea of Matsuda et al.'s technique is that for a function

$$
\text { get }:: \tau_{1} \rightarrow \tau_{2}
$$

one finds a function

$$
\text { compl :: } \tau_{1} \rightarrow \tau_{3}
$$

such that the pairing of the two,

$$
\begin{aligned}
& \text { paired }:: \tau_{1} \rightarrow\left(\tau_{2}, \tau_{3}\right) \\
& \text { paired } s=(\text { get } s, \text { compl } s)
\end{aligned}
$$

is an injective function. Given a "partial inverse" inv :: $\left(\tau_{2}, \tau_{3}\right) \rightarrow$ Maybe $\tau_{1}$ of paired, satisfying the requirements that

- for every $s:: \tau_{1}$,

$$
\text { inv }(\text { paired } s) \equiv \text { Just } s \text {, }
$$

and

- for every $s^{\prime}:: \tau_{1}, v^{\prime}:: \tau_{2}$, and $c:: \tau_{3}$, if inv $\left(v^{\prime}, c\right) \equiv$ Just $s^{\prime}$, then

$$
\text { paired } s^{\prime} \equiv\left(v^{\prime}, c\right) \text {, }
$$

one obtains that

$$
\begin{aligned}
& \text { put }:: \tau_{1} \rightarrow \tau_{2} \rightarrow \text { Maybe } \tau_{1} \\
& \text { puts } v^{\prime}=\operatorname{inv}\left(v^{\prime}, \text { compls }\right)
\end{aligned}
$$

is consistent for get.

The approach of Matsuda et al. (2007) is to perform all the above by syntactic program transformations. For a certain class of programs, they give an algorithm that automatically derives compl from get in such a way that paired is indeed injective. Then instead of the definition for paired above they produce one using a tupling transformation (Pettorossi 1977) that avoids the two independent traversals of $s$ with get and compl. They syntactically invert paired to obtain inv (though they make inv implicitly a partial function, not explicitly in the type as above), and subsequently fuse the computations of inv and compl in the definition of put, again using a syntactic transformation (Wadler 1990).

We illustrate the syntactic approach based on our two running examples.

Running Example 1 (syntactically). The function alluded to in the first running example, sieving a list to keep only every second element, could have been defined as follows:

$$
\begin{aligned}
& \operatorname{get}_{1}::[\alpha] \rightarrow[\alpha] \\
& \operatorname{get}_{1}[]=[] \\
& \operatorname{get}_{1}[x]=[] \\
& \operatorname{get}_{1}(x: y: z s)=y:\left(\operatorname{get}_{1} z s\right)
\end{aligned}
$$


That function definition fulfills the syntactic prerequisites imposed by Matsuda et al. They are (necessary 8 and sufficient): that functions must be first-order, must be affine (i.e., no variable occurs more than once in a single right-hand side), and that there must be no function call with anything else than variables in its arguments.

Given the above function definition, the following complement function is automatically derived 9

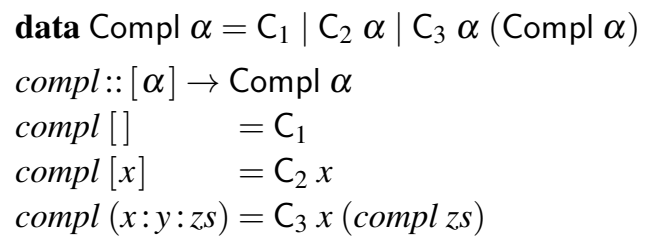

Tupling of get $_{1}$ and compl gives the following definition for the paired function:

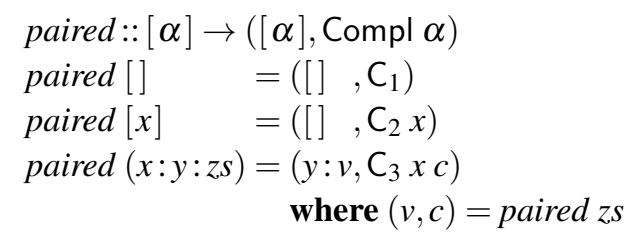

Syntactic inversion, (here) basically just exchanging left- and right-hand sides, plus introduction of monadic error propagation, gives:

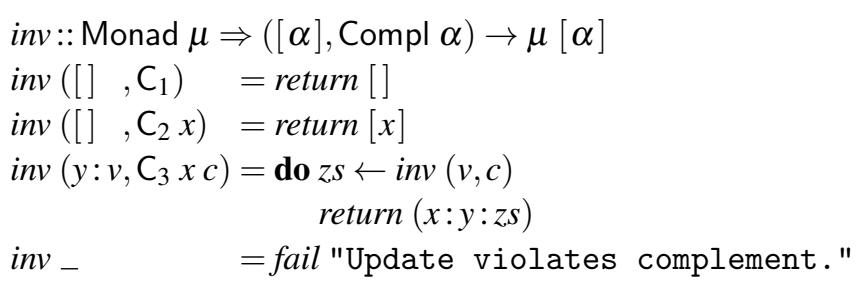

Finally,

$$
\begin{aligned}
& \text { put }:: \text { Monad } \mu \Rightarrow[\alpha] \rightarrow[\alpha] \rightarrow \mu[\alpha] \\
& \text { put } s v^{\prime}=\operatorname{inv}\left(v^{\prime}, \text { compl } s\right)
\end{aligned}
$$

can be fused to:

$$
\begin{aligned}
& \text { put :: Monad } \mu \Rightarrow[\alpha] \rightarrow[\alpha] \rightarrow \mu[\alpha] \\
& \text { put }[] \quad[] \quad=\text { return }[] \\
& \text { put }[x] \quad[] \quad=\text { return }[x] \\
& \text { put }(x: y: z s)\left(y^{\prime}: v^{\prime}\right)=\mathbf{d o} z s^{\prime} \leftarrow \text { put } z s v^{\prime} \\
& \text { return }\left(x: y^{\prime}: z s^{\prime}\right) \\
& \text { put__ _ _ = fail "Update violates complement." }
\end{aligned}
$$


Note that, just as was the case for the original semantic bidirectionalization technique here, put $s v^{\prime}$ fails if and only if length $\left(\right.$ get $\left._{1} s\right) \neq$ length $v^{\prime}$. Indeed, bff get ${ }_{1}$ (from Example 1 in Section 3 and the above put are semantically equivalent (at type $[\tau] \rightarrow[\tau] \rightarrow$ Maybe $[\tau]$, for $\tau$ that is an instance of $\mathrm{Eq}$ ).

Running Example2 2 (syntactically). The function alluded to in the second running example, keeping every element of a list except for the last one, could have been defined as follows 10

$$
\begin{aligned}
& \operatorname{get}_{2}::[\alpha] \rightarrow[\alpha] \\
& \operatorname{get}_{2}[] \quad=[] \\
& \operatorname{get}_{2}[x]=[] \\
& \operatorname{get}_{2}(x: y: z s)=x:\left(\text { get }^{\prime} y z s\right) \\
& \operatorname{get}^{\prime}:: \alpha \rightarrow[\alpha] \rightarrow[\alpha] \\
& \operatorname{get}^{\prime} x[]=[] \\
& \operatorname{get}^{\prime} x(y: z s)=x:\left(\text { get }^{\prime} y z s\right)
\end{aligned}
$$

For that function definition, the syntactic approach produces the following complement function:

$$
\begin{aligned}
& \text { data Compl } \alpha=C_{1}\left|C_{2} \alpha\right| C_{3} \alpha \\
& \operatorname{compl}::[\alpha] \rightarrow \text { Compl } \alpha \\
& \operatorname{compl}[] \quad=C_{1} \\
& \operatorname{compl}[x] \quad=C_{2} x \\
& \operatorname{compl}(x: y: z s)=\operatorname{compl}^{\prime} y z s \\
& \operatorname{compl}:: \alpha \rightarrow[\alpha] \rightarrow \operatorname{Compl} \alpha \\
& \operatorname{compl} x[]=C_{3} x \\
& \operatorname{compl}^{\prime} x(y: z s)=\operatorname{compl}^{\prime} y z s
\end{aligned}
$$

Tupling, inversion, and fusion (not spelled out here in detail) ultimately give functions put and (helper) put' such that put $s v^{\prime}$ succeeds if and only if length $\left(\right.$ get $\left._{2} s\right)$ and length $v^{\prime}$ are equal or both greater than zero. In contrast, we have seen in Section 3 that the original semantic bidirectionalization technique (again) allows no view updates that change the shape here, i.e., bff get ${ }_{2} s v^{\prime}$ is only successful if length $\left(\right.$ get $\left._{2} s\right)=$ length $v^{\prime}$. A few representative calls and their results will be given later in Table 1

From the examples considered, we see that the syntactic bidirectionalization technique of Matsuda et al. (2007) and the original (non-plugged) semantic bidirectionalization technique of Voigtländer (2009) can agree or disagree in terms of updateability. Actually, it seems that for programs that can be handled by both, the syntactic technique on its own is never worse than the original semantic technique on its own. Interestingly, the method of choice for improvement over both, proposed by Voigtländer et al. (2010) and recollected in this paper, is to defer the syntactic technique to the role of a plug-in (basically as a black box), with the technique of Voigtländer (2009) in the master role.

10 A helper function get' is used to prevent a function call with an argument that is not a variable. 
Specifically, for functions get that are polymorphic and at the same time satisfy the syntactic restrictions imposed by Matsuda et al.'s technique, we can use that technique for deriving sput from an explicit syntactic definition for a function sget (independently of, but derived from, get) that is semantically equivalent to shapify get.

Running Example 1 (with syntactic technique as plug-in). We have seen above in the current subsection and in Section 3 that for the function get $_{1}$ in question both syntactic and (the original version of) semantic bidirectionalization on their own lead to quite limited updateability: both put $s v^{\prime}$ and bff get $s v^{\prime}$ only succeed if length (get $\left.t_{1} s\right)=$ length $v^{\prime}$.

On the other hand, by combining the two techniques, we can proceed as follows. The sget "corresponding to" get $_{1}$, as obtained via a straightforward syntactic transformation from the definition of get $_{1}$ given earlier in this subsection, looks as follows:

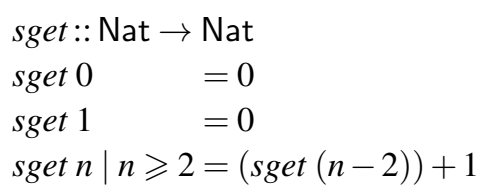

For it, the syntactic bidirectionalization method of Matsuda et al. (2007) produces the following complement function:

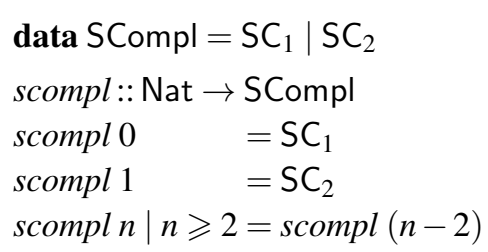

Note that the move from $[\alpha]$ to Nat in get $_{1} \mapsto$ sget has made the complement function much simpler: no collection of any variables (as was necessary in the definition of compl, to make up for the dropping of variables in the definition of get $_{1}$ ), and no constructor around the recursive call. (All this, thanks to explicit optimization effort embedded in Matsuda et al.'s transformation to "make the complement smaller".) The advantage is that a simpler/smaller complement function means better updateability of the ultimately obtained put-function. Here, tupling, inversion, and fusion give:

$$
\begin{aligned}
\text { sput }:: \mathrm{Nat} \rightarrow \mathrm{Nat} & \rightarrow \text { Maybe Nat } \\
\text { sput } 00 & =\text { return } 0 \\
\text { sput } 10 \quad & =\text { return } 1 \\
\text { sput } l_{s} 0 \mid l_{s} \geqslant 2 & =\text { sput }\left(l_{s}-2\right) 0 \\
\text { sput } l_{s} l_{v^{\prime}} \mid l_{v^{\prime}} \geqslant 1 & =\text { do } l_{s^{\prime}} \leftarrow \operatorname{sput} l_{s}\left(l_{v^{\prime}}-1\right) \\
& \text { return }\left(l_{s^{\prime}}+2\right)
\end{aligned}
$$

which is equivalent to the (desirable) sput-function provided for get $_{1}$ "by hand" in Section 5.1. So by using bff plug get $_{1}$ sput, or dbff get ${ }_{1}$ sput ', ', with this sput we enjoy good and intuitive updateability, without requiring manual intervention.

Running Example 2 (with syntactic technique as plug-in). We have seen further above in the current subsection and in Section 3 that for the function get $_{2}$ in question the updateability achieved by syntactic bidirectionalization is that put $s v^{\prime}$ succeeds whenever 
length $\left(\right.$ get $\left._{2} s\right)$ and length $v^{\prime}$ are equal or both greater than zero, while the original semantic technique is only successful if length $\left(\right.$ get $\left._{2} s\right)=$ length $v^{\prime}$. Let us analyze how the combination of the two techniques fares.

The move from $[\alpha]$ to Nat yields:

$$
\begin{aligned}
& \text { sget }:: \text { Nat } \rightarrow \text { Nat } \\
& \text { sget } 0 \quad=0 \\
& \text { sget } 1 \quad=0 \\
& \text { sget } n \mid n \geqslant 2=\left(\text { sget }^{\prime}(n-2)\right)+1 \\
& \text { sget }^{\prime}:: \text { Nat } \rightarrow \text { Nat } \\
& \text { sget }^{\prime} 0 \quad=0 \\
& \text { sget }^{\prime} n \mid n \geqslant 1=\left(\text { sget }^{\prime}(n-1)\right)+1
\end{aligned}
$$

Note that regarding the helper function $g e t^{\prime}$ (from earlier in this subsection) one argument becomes superfluous. Indeed, when moving from $[\alpha]$ to Nat, there is no role to play anymore for content elements of type $\alpha$.

The automatic view complement generation of Matsuda et al. (2007) yields either of two functions scompl $_{1} /$ scompl $_{2}$ for sget (with data SCompl $=\mathrm{SC}_{1}\left|\mathrm{SC}_{2}\right| \mathrm{SC}_{3}$ ) which differ only in their last defining equation:

$$
\begin{array}{ll}
\operatorname{scompl}_{1}:: \mathrm{Nat} \rightarrow & \mathrm{SCompl} \\
\operatorname{scompl}_{1} 0 & =\mathrm{SC}_{1} \\
\operatorname{scompl}_{1} 1 & =\mathrm{SC}_{2} \\
\operatorname{scompl}_{1} n \mid n \geqslant 2 & =\mathrm{SC}_{1}
\end{array}
$$

and

$$
\begin{array}{ll}
\text { scompl }_{2}:: \text { Nat } & \rightarrow \text { SCompl } \\
\text { scompl }_{2} 0 & =\mathrm{SC}_{1} \\
\text { scompl }_{2} 1 & =\mathrm{SC}_{2} \\
\text { scompl }_{2} n \mid n \geqslant 2 & =\mathrm{SC}_{2}
\end{array}
$$

while for $s g e t$, one obtains the following complement function:

$$
\begin{aligned}
& \text { scompl }^{\prime}:: \mathrm{Nat} \rightarrow \mathrm{SCompl} \\
& \text { scompl }^{\prime} 0=\mathrm{SC}_{3} \\
& \text { scompl }^{\prime} n \mid n \geqslant 1=\mathrm{SC}_{3}
\end{aligned}
$$

Tupling, inversion, and fusion ultimately give two choices $s p u t_{1}$ and $s p u t_{2}$, for $s c o m p l_{1}$ and scompl $_{2}$. Let us compare the results of combining syntactic and semantic bidirectionalization, i.e. the now two possible functions $d b f f g_{2} t_{2} s u t_{1}$ and $d b f f g t_{2} s p u t_{2}$, to the results of either only (the original version of) semantic or only syntactic bidirectionalization, i.e. to $b f f g_{2}$ à la Section 3 and to $p u t$ from the continuation of Example 2 further above in the current subsection. Table 1 shows a few representative calls and their results, where $d p u t_{1} \equiv d b f f$ get $_{2}$ sput $_{1}$ and $d p u t_{2} \equiv d b f f$ get $_{2}$ sput $_{2}$. By our coarse measure, comparing the sizes of the applicability domains of put-functions, the combined technique is better than either of the two original techniques. However, some skepticism is appropriate regarding results like those for $s=" \mathrm{ab} "$ and $v^{\prime}=$ "xy" here: all put-functions except the one obtained by the purely semantic technique map that $\left(s, v^{\prime}\right)$ pair to some $s^{\prime}$, but the 
Table 1. Comparing bidirectionalization methods for the get-function from Example 2

\begin{tabular}{|c|c|c|c|c|c|c|}
\hline \multirow[b]{2}{*}{$s$} & \multirow[b]{2}{*}{ get $_{2} s$} & \multirow[b]{2}{*}{$v^{\prime}$} & \multirow{2}{*}{$\frac{\text { semantic }}{b f f \operatorname{get}_{2} s v^{\prime}}$} & \multirow{2}{*}{$\frac{\text { syntactic }}{\text { put } s v^{\prime}}$} & \multicolumn{2}{|c|}{ combined } \\
\hline & & & & & $d p u t_{1}{ }^{\prime}, ' s v^{\prime}$ & $d^{\prime} u t_{2}{ }^{\prime}, ' s v^{\prime}$ \\
\hline " " & " " & " " & Just " " & Just " " & Just " " & Just " " \\
\hline " " & " " & "x" & Nothing & Nothing & Just "x " & Nothing \\
\hline " " & " " & "xy" & Nothing & Nothing & Just "xy " & Nothing \\
\hline "a" & " " & " " & Just "a" & Just "a" & Just "a" & Just "a" \\
\hline "a" & " " & "x" & Notl & Nothing & Nothing & Just "x " \\
\hline "ab" & "a" & " " & Not & Nothing & Just & Just " " \\
\hline "ab" & "a" & "x" & Just "xb" & Just "xb" & Just "xb" & Just "xb" \\
\hline "ab" & "a" & "xy" & Noth & Just "xyb" & Just "xy " & Just "xy " \\
\hline "abc" & "ab" & " " & Noth & Nothing & Just " " & Just " " \\
\hline "abc" & "ab" & "x" & Notl & Just "xc" & Just "x " & Just "x " \\
\hline "abc" & "ab" & "xy" & Just "xyc" & Just "xyc" & Just "xyc" & Just "xyc" \\
\hline "abc" & "ab" & "xyz" & Nothing & Just "xyzc" & Just "xyz " & Just "xyz " \\
\hline
\end{tabular}

put-function obtained using the purely syntactic technique certainly makes the best choice concerning what that $s^{\prime}$ should be. We discuss this aspect further in the next section 11

\section{Explicit Bias}

Through the numbering scheme of our "template sources" via $[1 \ldots n]$ for a concrete source of length $n$, there is a certain bias that manifests itself when an update changes the length of the view. For example, while it is nice that in the continuation of Example 2 in Section 5.1 (and also in Section 5.2, and similarly for $d p u t_{1}$ in Section 5.3. Table 1) we have

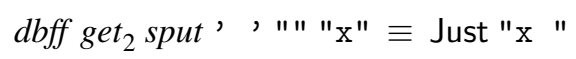

and

$$
\text { dbff get }_{2} \text { sput }, \quad, " \text { " "xy" } \equiv \text { Just "xy " }
$$

(in contrast to the completely semantically obtained bff get $_{2}$ and the completely syntactically obtained put, which both give Nothing in both cases; cf. Table 1), it is disappointing that

$$
\text { dbff } \text { get }_{2} \text { sput , , "ab" "xy" } \equiv \text { Just "xy " }
$$

(instead of Just "xyb"). The reason for this is simple: the use of $[1 \ldots n]$ and $\left[1 \ldots n^{\prime}\right]$ in the definition of $b f f_{\text {plug }}$ in Section 4.4 means that when the updated source becomes shorter than the original source, then it's the elements towards the rear of the original source that become discarded; while if the updated source becomes longer, then again positions towards the rear of the new source will be considered to be "additional" and thus will be filled with the default value. So there is an implicit assumption that shape-changing updates

11 Orthogonally, there would be more to say, and is said by Voigtländer et al. 2010), about updateability solely in terms of applicability domains. In particular, Section 7 of that paper contains examples showing how by involving additional syntactic transformations (Giesl 2000. Giesl et al. 2007) one can extend applicability further. Note also that, by virtue of the plugin approach, we will directly profit from further (independent) improvements of the syntactic bidirectionalization technique itself. 
will always happen in such a way that the corresponding insertions or deletions affect the end of the source list, rather than its front or other elements.

There is an easy remedy for the observed phenomenon. If we simply replace the lines

$$
\text { let } t=[1 \ldots n]
$$

and

$$
\text { let } t=\left[1 \ldots n^{\prime}\right]
$$

in the definition of $b f f_{\text {plug }}$ by

$$
\text { let } t=\text { reverse }[1 \ldots n]
$$

and

$$
\text { let } t=\text { reverse }\left[1 \ldots n^{\prime}\right]
$$

respectively, then Theorems 5 and 6 - and thus Corollaries 5 and 6 - continue to hold,

\begin{tabular}{|c|c|c|c|}
\hline$S$ & $\operatorname{get}_{2} s$ & $v^{\prime}$ & ${\text { dbff } \text { get }_{2} \text { sput }}{ }^{\prime}, s v^{\prime}$ \\
\hline " " & " " & "x" & Just "x " \\
\hline " " & $" 1 "$ & "xy" & Just "xy " \\
\hline "a" & " " & "x" & Just "xa" \\
\hline "ab" & "a" & $" 1 "$ & Just "b" \\
\hline "ab" & "a" & "xy" & Just "xyb" \\
\hline "abc" & "ab" & $" 1 "$ & Just "c" \\
\hline "abc" & "ab" & "x" & Just "xc" \\
\hline "abc" & "ab" & "xyz" & Just "xyzc" \\
\hline
\end{tabular}
but instead of a rear update (insertion/deletion) bias, there is now a front update bias.

For example, the table in the continuation of Example 2 in Section 5.1 (the interesting subset thereof; all other entries remain unchanged) now becomes:

The entries that have changed are shaded above. Only where no (last element) value from the original source list is available do we still use a default value in the updated source. One could argue that in this specific case all the changes are for the better, but in general it is desirable to be able to influence what bias is used.

Making the bias explicit, and thus putting it under the potential control of the user, is easily possible by defining a further variation of $b f f_{\text {plug }}$ :

$$
\begin{aligned}
& \text { type Bias }=\mathrm{Nat} \rightarrow[\mathrm{Nat}] \\
& b_{f f} \text { bias }:: \text { Monad } \mu \Rightarrow(\forall \alpha .[\alpha] \rightarrow[\alpha]) \rightarrow(\text { Nat } \rightarrow \text { Nat } \rightarrow \text { Maybe Nat }) \\
& \rightarrow \text { Bias } \rightarrow(\forall \alpha \cdot[\alpha] \rightarrow[\alpha] \rightarrow \mu[\text { Maybe } \alpha]) \\
& \text { bff bias } g \text { get sput bias } s v^{\prime}=\text { do let } n=\text { length } s \\
& \text { let } t=\text { bias } n \\
& \text { let } g=\text { NatMap.fromDistinctList (zip } t \text { s) } \\
& \text { let } g^{\prime}=\text { foldr NatMap.delete } g(\text { get } t) \\
& n^{\prime} \leftarrow \text { case sput } n\left(\text { length } v^{\prime}\right) \text { of } \\
& \text { Nothing } \rightarrow \text { fail "..." } \\
& \text { Just } n^{\prime} \rightarrow \text { return } n^{\prime} \\
& \text { let } t=\operatorname{bias} n^{\prime} \\
& \text { let } h=\text { NatMap.fromDistinctList }\left(\text { zip }(\text { get } t) v^{\prime}\right)
\end{aligned}
$$




$$
\begin{aligned}
& \text { let } h^{\prime}=\text { NatMap.union } h g^{\prime} \\
& \text { return } \left.\left(\text { map (flip NatMap.lookup } h^{\prime}\right) t\right)
\end{aligned}
$$

as well as:

$$
\begin{aligned}
\text { bdbff :: Monad } \mu \Rightarrow(\forall \alpha .[\alpha] & \rightarrow[\alpha]) \rightarrow(\text { Nat } \rightarrow \text { Nat } \rightarrow \text { Maybe Nat }) \\
\rightarrow \text { Bias } & \rightarrow(\forall \alpha . \alpha \rightarrow[\alpha] \rightarrow[\alpha] \rightarrow \mu[\alpha]) \\
\text { bdbff get sput bias } d s v^{\prime}=\text { do } s^{\prime} \leftarrow \text { bff bias } \text { get sput bias } s v^{\prime} & \\
& \text { return }\left(\text { map }(\lambda \text { case }\{\text { Nothing } \rightarrow d ; \text { Just } y \rightarrow y\}) s^{\prime}\right)
\end{aligned}
$$

The only formal requirement we impose on a proper bias :: Bias, ensuring that analogues of Theorems 5 and 6 and of Corollaries 5 and 6 continue to hold, is that for every $n::$ Nat, bias $n$ should return a list that is a permutation of $[1 \ldots n]$. Then, we in particular obtain the following two corollaries.

Corollary 7. Let get:: $[\alpha] \rightarrow[\alpha]$ be semantically affine. Let sput be consistent for shapify get. Let bias :: Bias be proper (in the way just described). For every type $\tau$ and $d:: \tau$,

$$
\text { bdbff get sput bias } d::[\tau] \rightarrow[\tau] \rightarrow \text { Maybe }[\tau]
$$

is consistent for get $::[\tau] \rightarrow[\tau]$.

Corollary 8. Let get:: $[\alpha] \rightarrow[\alpha]$ be semantically affine. Let sput be consistent for shapify get. Let bias:: Bias be proper. For every type $\tau$ and $d:: \tau$,

$$
\text { bdbff get sput bias } d::[\tau] \rightarrow[\tau] \rightarrow \text { Maybe }[\tau]
$$

is fixed-shape-friendly for get $::[\tau] \rightarrow[\tau]$. (Moreover, the default value $d$ is not actually used in bdbff get sput bias $d s v^{\prime}$ if length (get $\left.s\right)=$ length $v^{\prime}$.)

Some good examples for bias are:

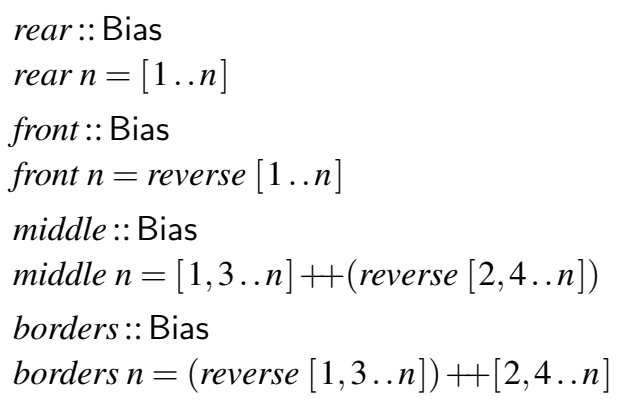

Some examples for the get-function from Example 1 (with sput as given in Section 5.1 and automatically obtained in Section 5.3, illustrating the effects of different bias strate-

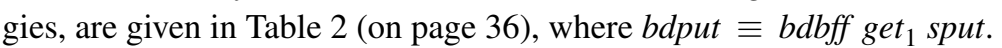

The beneficial effects, still for the case of the get-function from Example 1, might become even more apparent when also looking at cases where the data values in the source and view lists are not disjoint, as in Table 3 (on page 37). The simple hints about which bias to apply when reflecting specific updated views back to the source level are quite effective. 


\section{J. Voigtländer et al.}

\section{Going Generic}

Up to here, we have only considered the case of lists, by applying bidirectionalization to functions get $::[\alpha] \rightarrow[\alpha]$. On the other hand, both the syntactic bidirectionalization technique of Matsuda et al. (2007) and the original (non-plugged) semantic bidirectionalization technique of Voigtländer(2009) are already able to work on other data structures than lists. In this section, we catch up to such a more generic setting, by showing how $b f f_{\text {plug }}$ can be made suitably polymorphic over the type constructors on the input and output sides of get-functions. We do this in a similar way as the reformulation by Foster et al. (2012, Section 5.4) of the generic version of the original semantic bidirectionalization technique, namely by using explicit separations of data structures into their shape and content aspects, in the spirit of the shape calculus (Jay| 1995) and container representations (Abbott et al. 2003). Specifically, we start by introducing an abstraction for types that can hold shapes of members of other types, in the sense in which Nat has served as the type of shapes for lists. The way we set this up here is as a type class whose only operation is one that tells us how many element positions are associated with a given shape:

$$
\begin{gathered}
\text { class Shape } T \sigma \text { where } \\
\text { arity }: \sigma \rightarrow \mathrm{Nat}
\end{gathered}
$$

For example, for the shape type Nat the number of positions is the given natural number itself:

\section{instance ShapeT Nat where arity $n=n$}

Given a shape type, we can express that some data structure type is shaped accordingly, by providing functions for separating a data structure into shape and content and for reassembling a data structure from shape and content. The interface is as follows ${ }^{12}$

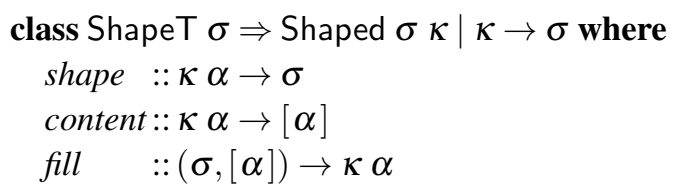

and we expect some natural laws to hold. The notion of position numbers must be consistent with how many content elements are actually extracted from a data structure of a given shape: arity $($ shape $x)=$ length (content $x$ ). The separation of a data structure into shape and content must be faithful, i.e., reassembly must be possible: fill (shape $x$, content $x$ ) $\equiv x$. Moreover, if a data structure is put together from some shape and some content, then each of the two aspects must be respected: shape $(f i l l(s h, l)) \equiv \operatorname{sh}$ and content $(f i l l(s h, l)) \equiv l$. Of course, the latter two properties can only be expected if $s h$ and $l$ actually fit together, i.e., if arity $s h=$ length $l$. Indeed, we will only ever apply fill to such $(s h, l)$-combinations.

12 The functional dependency annotation " $\mid \kappa \rightarrow \sigma$ " serves to resolve ambiguities and reduce the need for type annotations. It imposes the constraint that the data structure type always uniquely determines the underlying shape type, so that in particular, the output type of any application shape $x$ already follows from the type of $x$. 
To describe that lists are shaped over Nat, in precisely the way used in this paper so far, we can simply express that the shape of a list is its length, its content is itself, and reassembly is equally straightforward:

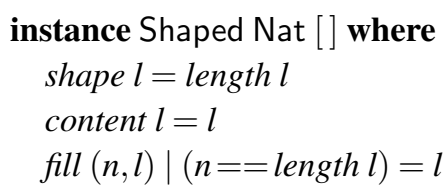

It is easy to see that the laws mentioned above all hold for this instance.

For the sake of an example for another data structure type than lists, consider the following data type definition:

$$
\text { data Tree } \alpha=\text { Leaf } \alpha \mid \text { Branch (Tree } \alpha)(\text { Tree } \alpha)
$$

One possibility for expressing the shape of a tree is to use a simpler tree with all content elements erased, or rather overwritten with a trivial element. Using the unit type (), whose only value is also denoted by (), the following ShapeT instance makes available this notion of shape tree, along with the appropriate arity-function:

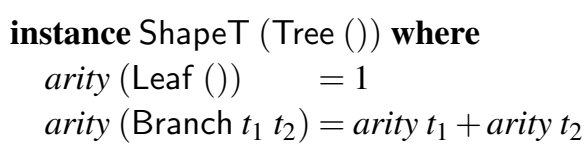

The other operations are also relatively straightforward recursive traversals:

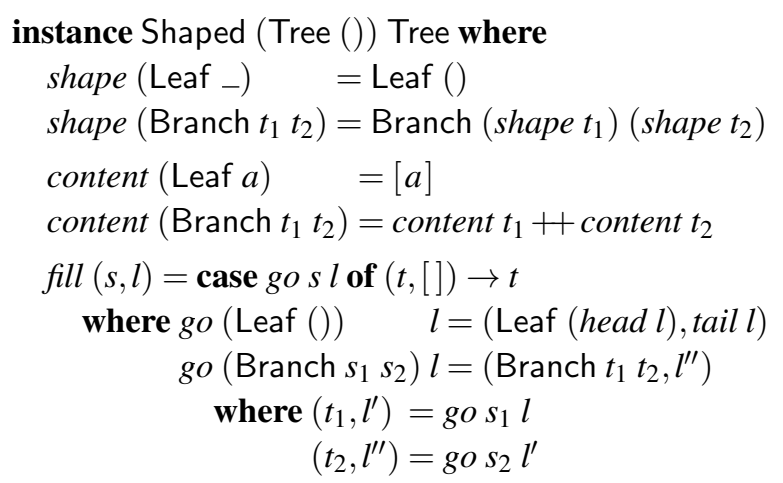

One can again establish that the required laws all hold. More generally, it is possible to provide suitable instances of this kind (i.e., $\kappa()$ as shape type for some $\kappa$ ) for a whole range of traversable data structures in a generic fashion (Gibbons and Oliveira 2009).

Given the generic setup, we can now make our contribution from the previous sections data-type-polymorphic. Let us start with the shapify-function. It previously had the type shapify $::(\forall \alpha .[\alpha] \rightarrow[\alpha]) \rightarrow($ Nat $\rightarrow$ Nat $)$ and definition shapify get $n=$ length $($ get $[1 \ldots n])$. Now we can provide a generic version:

$$
\begin{aligned}
& \text { shapify :: (Shaped } \left.\sigma \kappa, \text { Shaped } \sigma^{\prime} \kappa^{\prime}\right) \Rightarrow\left(\forall \alpha . \kappa \alpha \rightarrow \kappa^{\prime} \alpha\right) \rightarrow\left(\sigma \rightarrow \sigma^{\prime}\right) \\
& \text { shapify get sh }=\text { shape }(\operatorname{get}(\text { fill }(\operatorname{sh},[1 \ldots(\text { arity sh })])))
\end{aligned}
$$

in which lists on the input and output sides of get have been replaced by some shaped types (type constructors $\kappa$ and $\kappa^{\prime}$ ) and as a result, instead of a function from Nat to Nat, 
we obtain a function between the corresponding shape types $\left(\sigma\right.$ and $\left.\sigma^{\prime}\right)$. For example, if get has type Tree $\alpha \rightarrow[\alpha]$, then shapify get has type Tree ()$\rightarrow$ Nat. The implementation of the generic shapify-function follows the same idea as the list-specific one, namely to apply get to a template structure built according to the given original shape (and with irrelevant content), and to extract the resulting shape at the end. But instead of doing so in an adhoc fashion by direct list construction and consumption, only the interface provided by ShapeT and Shaped is used. The same principle guides the implementation of a data-typepolymorphic version of $b f f_{\text {plug }}$ :

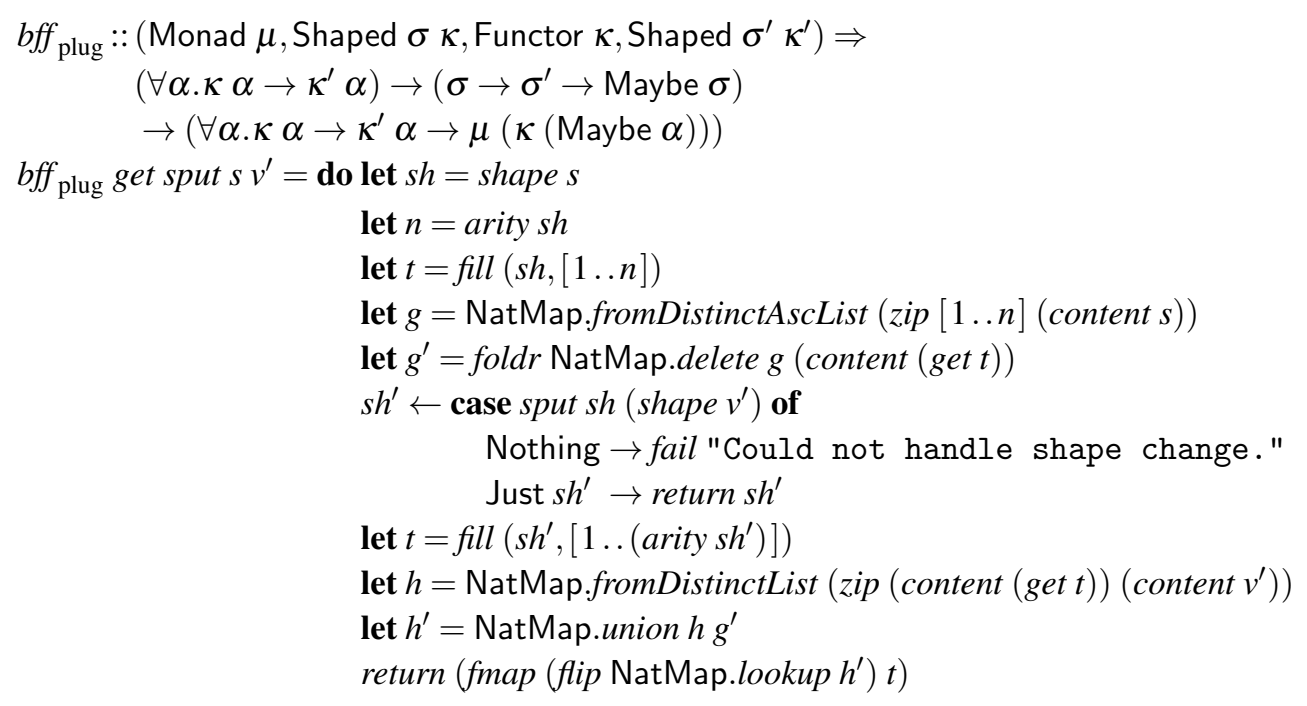

Instead of constructing a template $[1 \ldots n]$ from a list, we abstract a more general data structure to its shape, construct a template list from that, use it to "redecorate" the original data structure, and work from there, using the list of content items separately when constructing $g$. On the view side, we again work with the separation into content and shape, in particular constructing $g^{\prime}$ from the content of the outcome of the subcall to get (and we apply similar adaptations for constructing $h$ later on), and instead of applying sput to the lengths of lists, applying it to the shapes of $s$ and $v^{\prime}$. We create the second $t$, shadowing the first one, from the new shape (rather than just new list length), and in the end traverse it with fmap instead of the list-specific map-function. The latter explains why the type signature for $b f f_{\text {plug }}$ demands a suitable Functor instance for $\kappa$. The same is true in the case of $d b f f$, which now has type

$$
\begin{aligned}
d b f f:: & \left(\text { Monad } \mu \text {, Shaped } \sigma \kappa, \text { Functor } \kappa \text {, Shaped } \sigma^{\prime} \kappa^{\prime}\right) \Rightarrow \\
& \left(\forall \alpha . \kappa \alpha \rightarrow \kappa^{\prime} \alpha\right) \rightarrow\left(\sigma \rightarrow \sigma^{\prime} \rightarrow \text { Maybe } \sigma\right) \\
& \rightarrow\left(\forall \alpha . \alpha \rightarrow \kappa \alpha \rightarrow \kappa^{\prime} \alpha \rightarrow \mu(\kappa \alpha)\right)
\end{aligned}
$$

and definition exactly as before, just with map replaced by fmap.

It is easy to see (by comparing definitions) that the generic functions bff plug, shapify, and $d b f f$ reduce to their list-specific versions given earlier when accordingly applied, since with the ShapeT/Shaped-instances set up for the list case, essentially arity $\equiv i d$, shape $\equiv$ length, content $\equiv i d$, and fill $\equiv$ snd. Of course, the real worth is that now we can apply the functions at other types than lists as well. For example, we can use $b f f_{\text {plug }}$ or $d b f f$ for a get 
that operates on Trees, and call out to an sput derived using the technique of Matsuda et al. (2007) from sget semantically equivalent to shapify get (and thus simpler than get itself). The two examples we will instead be looking at here are a bit more mundane, but serve well to illustrate some interesting aspects regarding the generic setup we have established now.

Example 3. Assume we want to bidirectionalize the function length:: $[\alpha] \rightarrow$ Nat. It is a bit of an extreme case, since the output side has no content elements, instead only a monomorphic value. Nevertheless, the task should in principle be doable, and there are also some reasonable expectations what the bidirectionalized function might do (probably cutting off a source list at some point if the updated view is a natural number smaller than the original source list length, and extending the list appropriately in the opposite case).

A small technical problem is that the type $[\alpha] \rightarrow$ Nat cannot be directly seen as $\kappa \alpha \rightarrow$ $\kappa^{\prime} \alpha$ for some instances of $\kappa$ and $\kappa^{\prime}$, precisely because Nat is just a monomorphic type, not a polymorphic type constructor applied to $\alpha$. This is easily overcome, though, using an auxiliary type constructor definition:

\section{newtype Const $\alpha \beta=$ Const $\alpha$}

and then actually bidirectionalizing the following function:

$$
\begin{aligned}
& \text { get }_{3}::[\alpha] \rightarrow \text { Const Nat } \alpha \\
& \text { get }_{3}=\text { Const } \circ \text { length }
\end{aligned}
$$

Now the question is what shape type to introduce for the type constructor Const Nat, i.e., which $\sigma$ to choose such that one can give reasonable definitions of functions arity:: $\sigma \rightarrow$ Nat, shape :: Const Nat $\alpha \rightarrow \sigma$, content :: Const Nat $\alpha \rightarrow[\alpha]$, and fill :: $(\sigma,[\alpha]) \rightarrow$ Const Nat $\alpha$. It makes sense to consider $\sigma$ to be Nat itself, though we should be careful not to reuse the interpretation of Nat as shapes for lists from earlier on. After all, the situation is quite different here, as we can see by focusing on content. No value of a type Const Nat $\alpha$ can ever contain any $\alpha$-values, so content should never return a non-empty list here. Accordingly, arity should always return 0 , in contrast to arity $\equiv i d:: \mathrm{Nat} \rightarrow \mathrm{Nat}$ from the earlier instance. On the other hand, we cannot simply use a trivial type for $\sigma$, since the natural number stored in a value of a type Const Nat $\alpha$ must not be lost completely when separating shape and content. In fact, conceptually it should be preserved in the notion of shape. So the appropriate notion of shape here is a natural number, but it should be represented using a different (though isomorphic) type than Nat itself. One convenient way to proceed is to use the unit type () again, as follows:

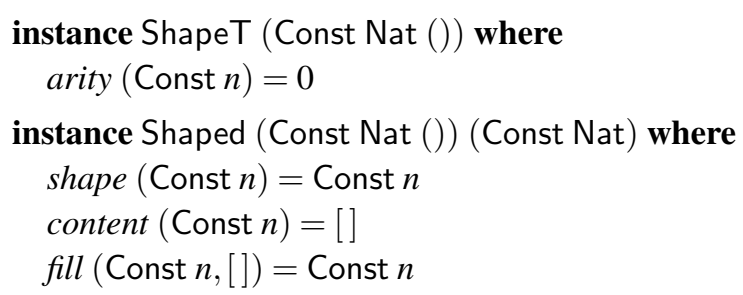

It is easy to see that the required laws all hold. 
What now about sget $\equiv$ shapify get $3::$ Nat $\rightarrow$ Const Nat ()? Since get $_{3}$ maps a list to its length, and the shape of a list is also its length, and the shape of a Const Nat $\alpha$ is simply the embedded natural number rewrapped with Const, we have that sget is simply Const as well, in particular it is injective and surjective! This makes the task of bidirectionalizing sget very simple since for forward functions that are injective and surjective, there is always only one appropriate backward function, namely the one which ignores the original source and applies the inverse of the forward function to the updated view. Here, this means:

$$
\begin{aligned}
& \text { sput }:: \text { Nat } \rightarrow \text { Const Nat }() \rightarrow \text { Maybe Nat } \\
& \text { sput _ (Const } n)=\text { Just } n
\end{aligned}
$$

Had we invested into a generic version of the search approach from Section 5.2 as well, we could even have avoided the manual provision of sput here, instead obtaining the same function simply as sput $t_{\text {search }}$ sget $^{13}$ One way or the other, we obtain

$$
\begin{aligned}
& \text { put }_{3}:: \text { Monad } \mu \Rightarrow[\alpha] \rightarrow \text { Const Nat } \alpha \rightarrow \mu[\text { Maybe } \alpha] \\
& \text { put }_{3}=\text { bff }_{\text {plug }} \text { get }_{3} \text { sput }
\end{aligned}
$$

that behaves exactly as desired. In fact, if we use a default value and encapsulate the Constwrapping for convenience:

$$
\begin{aligned}
& \text { put }:: \text { Monad } \mu \Rightarrow \alpha \rightarrow[\alpha] \rightarrow \text { Nat } \rightarrow \mu[\alpha] \\
& \text { put' } d s v^{\prime}=d b f f \text { get } \\
& 3 \text { sput } d s\left(\text { Const } v^{\prime}\right)
\end{aligned}
$$

we obtain results like these:

\begin{tabular}{ccc|c}
$s$ & get $_{3} s$ & $v^{\prime}$ & put ${ }^{\prime},{ }^{\prime} v^{\prime}$ \\
\hline "abc" & Const 3 & 2 & Just "ab" \\
"abc" & Const 3 & 4 & Just "abc "
\end{tabular}

Example 4. A well known "challenge problem" for bidirectionalization approaches is a function that takes a list of pairs and applies a projection to each pair, e.g., the function map snd :: $[(\alpha, \beta)] \rightarrow[\beta]$ in Haskell. If a list of $(\alpha, \beta)$-pairs is mapped to just the $\beta$ components, and the resulting list of $\beta$ s is shortened or extended, what should happen concerning the (superfluous or missing) $\alpha$ s? These shape changes are neither successfully handled by the syntactic bidirectionalization technique of Matsuda et al. (2007), nor by the non-plugged semantic bidirectionalization technique of Voigtländer (2009). Let us see how our new approach fares. (We will solve the shape-updatability, but not the more challenging aspect of potential realignment of $\alpha \mathrm{s}$ and $\beta \mathrm{s}$.)

At first glance, it might seem as if there is not much data-type-genericity to this example, since it is all about lists. However, the type $[(\alpha, \beta)]$ is actually more interesting. In particular, when abstracting it to a shape type, we cannot simply use a list length as notion of shape. Instead, when abstracting away the $\alpha$ s, any reasonable notion of shape must incorporate the $\beta \mathrm{s}$, and vice versa. To be able to express abstraction from just one of the two element types, we again need a bit of wrapping via an extra type constructor, like

13 Since sget is injective and surjective, a data-type-generic version of $s p u t_{\text {search }}$ working by enumeration of possible inputs would be guaranteed to succeed and end up with sput behaving exactly like the manually given one here. 
with Const in Example 3 We define:

$$
\text { newtype PairList } \alpha \beta=\text { PairList }[(\alpha, \beta)]
$$

and then consider:

$$
\begin{aligned}
& \text { get }_{4}:: \text { PairList } \alpha \beta \rightarrow[\beta] \\
& \text { get }_{4}(\text { PairList } l)=\text { map snd } l
\end{aligned}
$$

We need a shape type for the type constructor PairList $\alpha$ (since this will be $\kappa$ here, while $\kappa^{\prime}$ will be []). As motivated above, we need to preserve all $\alpha$ s, and indeed, the following are very natural definitions (and satisfy all required laws):

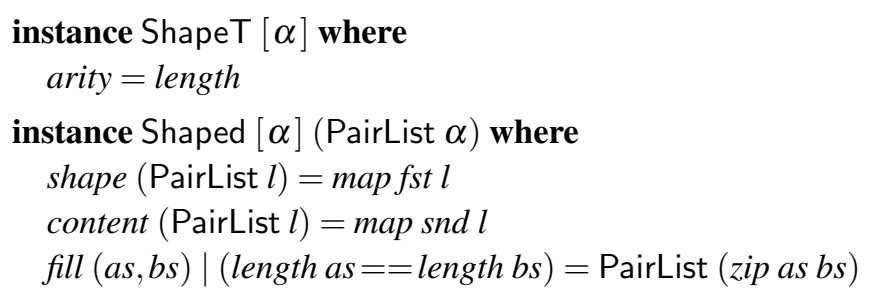

Due to the "Functor $\kappa \Rightarrow$ " constraint in the type of $b f f_{\text {plug }}(d b f f)$, we will also need a Functor instance for PairList $\alpha$ further below, and provide it as follows:

instance Functor (PairList $\alpha$ ) where

$$
\text { fmap } f(\text { PairList } l)=\text { PairList }(\operatorname{map}(\lambda(a, b) \rightarrow(a, f b)) l)
$$

Now let us take a look at sget $\equiv$ shapify get ${ }_{4}$. Conveniently, it has type $[\alpha] \rightarrow$ Nat, which is exactly the type dealt with in Example 3 In fact, more than that, shapify get $t_{4}$ is semantically equivalent to length as used in Example 3. That is, we can use put $d$ (for some $d$ ) from Example 3 as sput here, and obtain ${ }^{14}$

$$
\begin{aligned}
& \text { put } \left._{4}:: \text { Monad } \mu \Rightarrow \alpha \rightarrow \text { PairList } \alpha \beta \rightarrow[\beta] \rightarrow \mu \text { (PairList } \alpha \beta\right)
\end{aligned}
$$

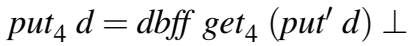

Another way to put this is that:

$$
\text { put } \left._{4} d=\operatorname{dbff}_{\text {get }}\left(\lambda s \rightarrow \text { dbff (Const } \circ \text { shapify get }{ }_{4}\right) \text { sput } d s \circ \text { Const }\right) \perp
$$

where sput is the one from Example 3 Given our observation that in Example 3 the sput could actually have been obtained as sput search $_{\text {(shapify get }}$ ), we could even write the above as:

$$
\begin{aligned}
& \text { put }_{4} d=\text { let } \text { get }_{3}=\text { Const } \circ \text { shapify get } 4 \\
& \text { in } \operatorname{dbff}_{\operatorname{get}}\left(\lambda s \rightarrow \operatorname{dbff}_{4} \operatorname{get}_{3}\left(\text { sput }_{\text {search }}\left(\text { shapify get }_{3}\right)\right) d s \circ \text { Const }\right) \perp
\end{aligned}
$$

Note that this working depends only on the type of $g t_{4}$. In particular, it does not depend on the observation from above that shapify get ${ }_{4}$ is semantically equivalent to length. The shape abstraction from get $_{4}$ to get $_{3} \equiv$ Const $\circ$ shapify get ${ }_{4}$ and then double use of $d b f f$, on the outer level for $\mathrm{get}_{4}$ and on the inner level for $\mathrm{get}_{3}$ defined in terms of $\mathrm{get}_{4}$, would have been

14 It so happens that the third argument of $d b f f$ will never be used here, so we choose $\perp$ as that default value. 
possible for other get $_{4}$-functions as well. In fact, besides the strategies from Sections 5.1 5.3 we could now add a fourth one: "5.4 Shape-Bidirectionalization by Bootstrapping",

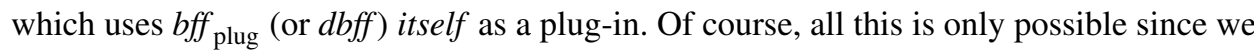
have provided a data-type-generic account of pluggable bidirectionalization. For otherwise, we would not have been able to use dbff both for a function and its shape-abstracted version.

Given the specific get $_{4}$-function above, we obtain results like these:

\begin{tabular}{|c|c|c|c|}
\hline$s$ & get $_{4}$ (PairList $\left.s\right)$ & $v^{\prime}$ & put $_{4}{ }^{\prime}, \quad$ (PairList $\left.s\right) v^{\prime}$ \\
\hline$z i p$ "ab" $[1,2]$ & {$[1,2]$} & [3] & Just (PairList [('a', 3)]) \\
\hline zip "ab" $[1,2]$ & {$[1,2]$} & {$[3,4,5]$} & Just (PairList $\left.\left[\left({ }^{\prime} a^{\prime}, 3\right),\left({ }^{\prime} b^{\prime}, 4\right),\left({ }^{\prime}, 5\right)\right]\right)$ \\
\hline
\end{tabular}

Finding good pragmatic bias strategies, as in Section 6 , for the case of non-lists is a possible topic for future work.

\section{Conclusion}

We have shown how to refactor the semantic bidirectionalization technique of Voigtländer (2009) in such a way that other techniques can be used as plug-ins. The key idea is to separate shape from content, thus simplifying the problem of explicit bidirectionalization by posing it only on the shape level (going from get to sget $\equiv$ shapify get). That way, for example, the existing syntactic bidirectionalization technique of Matsuda et al. (2007) can give far better results (in combination) than for the general problem (on its own). We have also developed a data-type-generic account. An interesting development is that we have moved automatic bidirectionalization towards more customizability by users/programmers, both in terms of choosing plug-ins and in terms of providing explicit bias. That brings the techniques closer in spirit to the domain-specific language approaches in the tradition of Foster et al. (2007).

Finally, a few more words about formal properties of get/put-pairs are in order. We have taken laws GetPut (1) and PutGet (2), in the form of Definition 1, as consistency conditions. So in the terminology of Foster et al. (2012), we have considered partial well-behaved lenses. The literature also knows PutPut:

$$
\text { put }\left(\text { put } s v^{\prime}\right) v^{\prime \prime} \equiv \text { put } s v^{\prime \prime},
$$

which as one interesting consequence together with GetPut implies undoability:

$$
\text { put }\left(\text { put } s v^{\prime}\right)(\text { get } s) \equiv s .
$$

Or, for partial put, both are required to hold whenever put $s v^{\prime}$ is defined. The technique of Matsuda et al. (2007) satisfies these two laws (thus producing partial very well-behaved lenses), by virtue of being based on the constant-complement approach of Bancilhon and Spyratos (1981). Although not explicitly proved by Voigtländer (2009), the same is true for his technique. In fact, it can be reformulated via the constant-complement approach as well (Foster et al. 2012). So the question is natural whether semantic bidirectionalization with plug-ins can also be so based, and satisfies PutPut and undoability as well. The answer is No, as invocations like dput, , "abcd" "x" $\equiv$ Just "ax" $\equiv d p u t$, , "abyd" "x" for Example 1 show, where $d p u t=d b f f$ get $_{1}$ sput (cf. the continuation of this example 
in Section 5.11. Clearly, there is no way that dput , , "ax" "bd" is both Just "abcd" and Just "abyd" as undoability would demand; instead: dput, , "ax" "bd" 三 Just "ab d". (PutPut fails for a similar reason.) Is that bad news? We think not: any method that successfully deals with insertion and deletion updates for a function like the get $_{1}$ under consideration here will have to give up PutPut and undoability. Indeed, these two properties are often considered undesirable, precisely because they significantly limit the transformations one can hope to deal with (Foster et al. 2007; Gottlob et al. 1988, Keller 1987).

\section{Acknowledgments}

We thank the anonymous reviewers of the earlier related paper (Voigtländer et al. 2010) and of the present article for their insightful comments and suggestions. Part of this work is supported by JSPS KAKENHI Grant Numbers 22800003 and 24700020. 
Table 2. Comparing bias strategies for our combined technique on the get-function from Example 1.

\begin{tabular}{|c|c|c|c|c|c|c|}
\hline$s$ & $\operatorname{get}_{1} s$ & $v^{\prime}$ & bdput rear' 's $s v^{\prime}$ & bdput front, , $s v^{\prime}$ & bdput middle, ' $s v^{\prime}$ & bdput borders, ', $s v^{\prime}$ \\
\hline "abcd" & "bd" & "x" & Just "ax" & Just "cx" & Just "ax" & Just " $\mathrm{x} "$ \\
\hline "abcd" & "bd" & "xyz" & Just "axcy z" & Just " xaycz" & Just "ax ycz" & Just " $\mathrm{x} y \mathrm{z} "$ \\
\hline "abcd" & "bd" & "xyzv" & Just "axcy $z$ v" & Just " $\mathrm{x}$ yazcv" & Just "ax y zcv" & Just " xaycz v" \\
\hline "abcde" & "bd" & "x" & Just "axc" & Just "cxe" & Just "axe" & Just " x " \\
\hline "abcde" & "bd" & "xyz" & Just "axcyez " & Just " xaycze" & Just "axcy ze" & Just " $\mathrm{x}$ y $z$ " \\
\hline "abcde" & "bd" & "xyzv" & Just "axcyez v " & Just " x yazcve" & Just "axcy z ve" & Just " xayczev " \\
\hline "abcde" & "bd" & "xyzvw" & Just "axcyez v w " & Just " x y zavcwe" & Just "axcy $z$ v we" & Just " $\mathrm{x}$ y $\mathrm{z}$ v $\mathrm{w}$ " \\
\hline
\end{tabular}


Table 3. More update bias examples for get $_{1}$ from Example 1

\begin{tabular}{cccc|l} 
bias & $s$ & get $_{1} s$ & $v^{\prime}$ & bdput bias , $s v^{\prime}$ \\
\hline rear & "abcd" & "bd" & "x" & Just "ax" \\
rear & "abcde" & "bd" & "x" & Just "axc" \\
front & "abcd" & "bd" & "x" & Just "cx" \\
front & "abcde" & "bd" & "x" & Just "cxe" \\
middle & "abcd" & "bd" & "x" & Just "ax" \\
middle & "abcde" & "bd" & "x" & Just "axe" \\
borders & "abcd" & "bd" & "x" & Just " x" \\
borders & "abcde" & "bd" & "x" & Just " x " \\
rear & "abcd" & "bd" & "bdx" & Just "abcd x" \\
rear & "abcd" & "bd" & "bdxy" & Just "abcd x y" \\
rear & "abcde" & "bd" & "bdx" & Just "abcdex " \\
rear & "abcde" & "bd" & "bdxy" & Just "abcdex y " \\
front & "abcd" & "bd" & "xbd" & Just " xabcd" \\
front & "abcd" & "bd" & "xybd" & Just " x yabcd" \\
front & "abcde" & "bd" & "xbd" & Just " xabcde" \\
front & "abcde" & "bd" & "xybd" & Just " x yabcde" \\
middle & "abcd" & "bd" & "bxd" & Just "ab xcd" \\
middle & "abcd" & "bd" & "bxyd" & Just "ab x ycd" \\
middle & "abcde" & "bd" & "bxd" & Just "abcx de" \\
middle & "abcde" & "bd" & "bxyd" & Just "abcx y de" \\
borders & "abcd" & "bd" & "xbdy" & Just " xabcd y" \\
borders & "abcde" & "bd" & "xbdy" & Just " xabcdey " \\
borders & "abcde" & "bd" & "xybdzv" & Just " x yabcdez v "
\end{tabular}


Bibliography

M. Abbott, T. Altenkirch, and N. Ghani. Categories of containers. In Foundations of Software Science and Computational Structures, Proceedings, volume 2620 of LNCS, pages 23-38. Springer-Verlag, 2003.

F. Bancilhon and N. Spyratos. Update semantics of relational views. ACM Transactions on Database Systems, 6(3):557-575, 1981.

A. Bohannon, B.C. Pierce, and J.A. Vaughan. Relational lenses: A language for updatable views. In Principles of Database Systems, Proceedings, pages 338-347. ACM Press, 2006.

A. Bohannon, J.N. Foster, B.C. Pierce, A. Pilkiewicz, and A. Schmitt. Boomerang: Resourceful lenses for string data. In Principles of Programming Languages, Proceedings, pages 407-419. ACM Press, 2008.

K. Czarnecki, J.N. Foster, Z. Hu, R. Lämmel, A. Schürr, and J.F. Terwilliger. Bidirectional transformations: A cross-discipline perspective. In International Conference on Model Transformation, Proceedings, volume 5563 of LNCS, pages 260-283. Springer-Verlag, 2009.

J.N. Foster, M.B. Greenwald, J.T. Moore, B.C. Pierce, and A. Schmitt. Combinators for bidirectional tree transformations: A linguistic approach to the view-update problem. ACM Transactions on Programming Languages and Systems, 29(3):17, 2007.

J.N. Foster, A. Pilkiewicz, and B.C. Pierce. Quotient lenses. In International Conference on Functional Programming, Proceedings, pages 383-395. ACM Press, 2008.

J.N. Foster, K. Matsuda, and J. Voigtländer. Three complementary approaches to bidirectional programming. In Spring School on Generic and Indexed Programming 2010, Revised Lectures, volume 7470 of LNCS, pages 1-46. Springer-Verlag, 2012.

J. Gibbons and B.C.d.S. Oliveira. The essence of the iterator pattern. Journal of Functional Programming, 19(3-4):377-402, 2009.

J. Giesl. Context-moving transformations for function verification. In Logic-Based Program Synthesis and Transformation 1999, Selected Papers, volume 1817 of LNCS, pages 293-312. Springer-Verlag, 2000.

J. Giesl, A. Kühnemann, and J. Voigtländer. Deaccumulation techniques for improving provability. Journal of Logic and Algebraic Programming, 71(2):79-113, 2007.

G. Gottlob, P. Paolini, and R. Zicari. Properties and update semantics of consistent views. ACM Transactions on Database Systems, 13(4):486-524, 1988.

S. Hidaka, Z. Hu, K. Inaba, H. Kato, K. Matsuda, and K. Nakano. Bidirectionalizing graph transformations. In International Conference on Functional Programming, Proceedings, pages 205-216. ACM Press, 2010.

Z. Hu, S.-C. Mu, and M. Takeichi. A programmable editor for developing structured documents based on bidirectional transformations. Higher-Order and Symbolic Computation, 21(1-2):89-118, 2008.

C.B. Jay. A semantics for shape. Science of Computer Programming, 25(2-3):251-283, 1995.

A.M. Keller. Comments on Bancilhon and Spyratos' "Update semantics and relational views". ACM Transactions on Database Systems, 12(3):521-523, 1987. 
K. Matsuda and M. Wang. Bidirectionalization for free with runtime recording. In Principles and Practice of Declarative Programming, Proceedings. ACM Press, 2013. To appear.

K. Matsuda, Z. Hu, K. Nakano, M. Hamana, and M. Takeichi. Bidirectionalization transformation based on automatic derivation of view complement functions. In International Conference on Functional Programming, Proceedings, pages 47-58. ACM Press, 2007.

K. Matsuda, Z. Hu, K. Nakano, M. Hamana, and M. Takeichi. Bidirectionalizing programs with duplication through complementary function derivation. Computer Software, 26(2): 56-75, 2009. In Japanese.

H. Pacheco and A. Cunha. Generic point-free lenses. In Mathematics of Program Construction, Proceedings, volume 6120 of LNCS, pages 331-352. Springer-Verlag, 2010.

H. Pacheco, A. Cunha, and Z. Hu. Delta lenses over inductive types. Electronic Communications of the European Association of Software Science and Technology, 49, 2012.

A. Pettorossi. Transformation of programs and use of tupling strategy. In Informatica, Proceedings, pages 1-6, 1977.

S.L. Peyton Jones, editor. Haskell 98 Language and Libraries: The Revised Report. Cambridge University Press, 2003.

J.C. Reynolds. Types, abstraction and parametric polymorphism. In Information Processing, Proceedings, pages 513-523. Elsevier, 1983.

C. Strachey. Fundamental concepts in programming languages. Lecture notes for a course at the International Summer School in Computer Programming, 1967. Reprint appeared in Higher-Order and Symbolic Computation, 13(1-2):11-49, 2000.

J. Voigtländer. Bidirectionalization for free! In Principles of Programming Languages, Proceedings, pages 165-176. ACM Press, 2009.

J. Voigtländer. Ideas for connecting inductive program synthesis and bidirectionalization. In Partial Evaluation and Program Manipulation, Proceedings, pages 39-42. ACM Press, 2012.

J. Voigtländer, Z. Hu, K. Matsuda, and M. Wang. Combining syntactic and semantic bidirectionalization. In International Conference on Functional Programming, Proceedings, pages 181-192. ACM Press, 2010.

P. Wadler. Theorems for free! In Functional Programming Languages and Computer Architecture, Proceedings, pages 347-359. ACM Press, 1989.

P. Wadler. Deforestation: Transforming programs to eliminate trees. Theoretical Computer Science, 73(2):231-248, 1990.

P. Wadler. The essence of functional programming (Invited talk). In Principles of Programming Languages, Proceedings, pages 1-14. ACM Press, 1992.

M. Wang, J. Gibbons, and N. Wu. Incremental updates for efficient bidirectional transformations. In International Conference on Functional Programming, Proceedings, pages 392-403. ACM Press, 2011.

M. Wang, J. Gibbons, K. Matsuda, and Z. Hu. Refactoring pattern matching. Science of Computer Programming, 2012. appeared online. 
